\title{
Double Lowering Operators on Polynomials
}

Paul TERWILLIGER

Department of Mathematics, University of Wisconsin, Madison, WI 53706-1388, USA

E-mail: terwilli@math.wisc.edu

Received September 15, 2020, in final form January 19, 2021; Published online January 28, 2021

https://doi.org/10.3842/SIGMA.2021.009

\begin{abstract}
Recently Sarah Bockting-Conrad introduced the double lowering operator $\psi$ for a tridiagonal pair. Motivated by $\psi$ we consider the following problem about polynomials. Let $\mathbb{F}$ denote an algebraically closed field. Let $x$ denote an indeterminate, and let $\mathbb{F}[x]$ denote the algebra consisting of the polynomials in $x$ that have all coefficients in $\mathbb{F}$. Let $N$ denote a positive integer or $\infty$. Let $\left\{a_{i}\right\}_{i=0}^{N-1},\left\{b_{i}\right\}_{i=0}^{N-1}$ denote scalars in $\mathbb{F}$ such that $\sum_{h=0}^{i-1} a_{h} \neq \sum_{h=0}^{i-1} b_{h}$ for $1 \leq i \leq N$. For $0 \leq i \leq N$ define polynomials $\tau_{i}, \eta_{i} \in \mathbb{F}[x]$ by $\tau_{i}=\prod_{h=0}^{i-1}\left(x-a_{h}\right)$ and $\eta_{i}=\prod_{h=0}^{i-1}\left(x-b_{h}\right)$. Let $V$ denote the subspace of $\mathbb{F}[x]$ spanned by $\left\{x^{i}\right\}_{i=0}^{N}$. An element $\psi \in \operatorname{End}(V)$ is called double lowering whenever $\psi \tau_{i} \in \mathbb{F} \tau_{i-1}$ and $\psi \eta_{i} \in \mathbb{F} \eta_{i-1}$ for $0 \leq i \leq N$, where $\tau_{-1}=0$ and $\eta_{-1}=0$. We give necessary and sufficient conditions on $\left\{a_{i}\right\}_{i=0}^{\bar{N}-1},\left\{b_{i}\right\}_{i=0}^{N-1}$ for there to exist a nonzero double lowering map. There are four families of solutions, which we describe in detail.
\end{abstract}

Key words: tridiagonal pair; $q$-exponential function; basic hypergeometric series; $q$-binomial theorem

2020 Mathematics Subject Classification: 33D15; 15A21

\section{Introduction}

This paper is mainly about polynomials and special functions, but in order to motivate things we first discuss a topic in linear algebra. The topic has to do with tridiagonal pairs [17] and their associated double lowering operator [7, 8, 9, 10]. A reader unfamiliar with tridiagonal pairs can safely skip to Section 2 . Let $V$ denote a vector space with finite positive dimension. A tridiagonal pair on $V$ is an ordered pair of linear maps $A: V \rightarrow V$ and $A^{*}: V \rightarrow V$ that satisfy the following conditions:

(i) each of $A, A^{*}$ is diagonalizable;

(ii) there exists an ordering $\left\{V_{i}\right\}_{i=0}^{d}$ of the eigenspaces of $A$ such that

$$
A^{*} V_{i} \subseteq V_{i-1}+V_{i}+V_{i+1}, \quad 0 \leq i \leq d,
$$

where $V_{-1}=0$ and $V_{d+1}=0$;

(iii) there exists an ordering $\left\{V_{i}^{*}\right\}_{i=0}^{\delta}$ of the eigenspaces of $A^{*}$ such that

$$
A V_{i}^{*} \subseteq V_{i-1}^{*}+V_{i}^{*}+V_{i+1}^{*}, \quad 0 \leq i \leq \delta,
$$

where $V_{-1}^{*}=0$ and $V_{\delta+1}^{*}=0$;

(iv) there does not exist a subspace $W \subseteq V$ such that $A W \subseteq W$ and $A^{*} W \subseteq W$ and $W \neq 0$ and $W \neq V$.

The tridiagonal pair concept originated in the theory of $Q$-polynomial distance-regular graphs [4, 11] where it is used to describe how the adjacency matrix is related to each dual adjacency matrix [17, Example 1.4], [29, Section 3]. Since that origin, the tridiagonal pair concept has 
found applications to all sorts of topics in special functions (orthogonal polynomials of the Askeyscheme [3, 25, 27, 32, 33], the Askey-Wilson algebra [14, 35, 40, 42]), Lie theory (the $\mathfrak{s l}_{2}$ loop algebra [19], the tetrahedron algebra [15, 21]), statistical mechanics (the Onsager algebra [12, 16] and $q$-Onsager algebra $[5,6,23,31,37,38]$ ), and quantum groups (the equitable presentation $[1,24,36]$, the quantum affine $\mathfrak{s l}_{2}$ algebra $[2,18,20,22], L$-operators [26, 39]). For more information about the above topics, see $[28,34]$ and the references therein.

Let $A, A^{*}$ denote a tridiagonal pair on $V$, as in the above definition. By [17, Lemma 4.5] the integers $d$ and $\delta$ from (ii), (iii) are equal; this common value is called the diameter of the pair. For $0 \leq i \leq d$ let $\theta_{i}$ (resp. $\theta_{i}^{*}$ ) denote the eigenvalue of $A$ (resp. $A^{*}$ ) for the eigenspace $V_{i}$ (resp. $V_{i}^{*}$ ). By [17, Theorem 11.1] the scalars

$$
\frac{\theta_{i-2}-\theta_{i+1}}{\theta_{i-1}-\theta_{i}}, \quad \frac{\theta_{i-2}^{*}-\theta_{i+1}^{*}}{\theta_{i-1}^{*}-\theta_{i}^{*}}
$$

are equal and independent of $i$ for $2 \leq i \leq d-1$. For this constraint the solutions can be given in closed form [17, Theorem 11.2]. The "most general" solution is called $q$-Racah, and will be described shortly.

By construction the vector space $V$ has a direct sum decomposition into the eigenspaces $\left\{V_{i}\right\}_{i=0}^{d}$ of $A$ and the eigenspaces $\left\{V_{i}^{*}\right\}_{i=0}^{d}$ of $A^{*}$. The vector space $V$ has two other direct sum decompositions of interest, called the first split decomposition $\left\{U_{i}\right\}_{i=0}^{d}$ and second split decomposition $\left\{U_{i}^{\Downarrow}\right\}_{i=0}^{d}$. By $[17$, Theorem 4.6] the first split decomposition satisfies

$$
\begin{aligned}
& U_{0}+U_{1}+\cdots+U_{i}=V_{0}^{*}+V_{1}^{*}+\cdots+V_{i}^{*}, \\
& U_{i}+U_{i+1}+\cdots+U_{d}=V_{i}+V_{i+1}+\cdots+V_{d}
\end{aligned}
$$

for $0 \leq i \leq d$. By $[17$, Theorem 4.6] the second split decomposition satisfies

$$
\begin{aligned}
& U_{0}^{\Downarrow}+U_{1}^{\Downarrow}+\cdots+U_{i}^{\Downarrow}=V_{0}^{*}+V_{1}^{*}+\cdots+V_{i}^{*}, \\
& U_{i}^{\Downarrow}+U_{i+1}^{\Downarrow}+\cdots+U_{d}^{\Downarrow}=V_{0}+V_{1}+\cdots+V_{d-i}
\end{aligned}
$$

for $0 \leq i \leq d$. By [17, Theorem 4.6],

$$
\begin{array}{ll}
\left(A-\theta_{i} I\right) U_{i} \subseteq U_{i+1}, & \left(A^{*}-\theta_{i}^{*} I\right) U_{i} \subseteq U_{i-1}, \\
\left(A-\theta_{d-i} I\right) U_{i}^{\Downarrow} \subseteq U_{i+1}^{\Downarrow}, & \left(A^{*}-\theta_{i}^{*} I\right) U_{i}^{\Downarrow} \subseteq U_{i-1}^{\Downarrow}
\end{array}
$$

for $0 \leq i \leq d$, where $U_{-1}=0, U_{d+1}=0$ and $U_{-1}^{\Downarrow}=0, U_{d+1}^{\Downarrow}=0$.

In [7, Sections 11 and 15] Sarah Bockting-Conrad introduces a linear map $\Psi: V \rightarrow V$ such that

$$
\Psi U_{i} \subseteq U_{i-1}, \quad \Psi U_{i}^{\Downarrow} \subseteq U_{i-1}^{\Downarrow}, \quad 0 \leq i \leq d .
$$

This map is called the double lowering operator or Bockting operator. In [7, Sections 9 and 15] Bockting-Conrad introduces an invertible linear map $\Delta: V \rightarrow V$ that commutes with $\Psi$ and sends $U_{i}$ onto $U_{i}^{\Downarrow}$ for $0 \leq i \leq d$. The maps $\Psi$ and $\Delta$ are related in the following way. For $0 \leq i \leq d$ define two polynomials

$$
\begin{aligned}
\tau_{i} & =\left(x-\theta_{0}\right)\left(x-\theta_{1}\right) \cdots\left(x-\theta_{i-1}\right), \\
\eta_{i} & =\left(x-\theta_{d}\right)\left(x-\theta_{d-1}\right) \cdots\left(x-\theta_{d-i+1}\right)
\end{aligned}
$$

in a variable $x$. Define the scalars

$$
\vartheta_{i}=\sum_{h=0}^{i-1} \frac{\theta_{h}-\theta_{d-h}}{\theta_{0}-\theta_{d}}, \quad 1 \leq i \leq d .
$$


By [7, Theorem 17.1],

$$
\Delta=\sum_{i=0}^{d} \frac{\eta_{i}\left(\theta_{0}\right)}{\vartheta_{1} \vartheta_{2} \cdots \vartheta_{i}} \Psi^{i}, \quad \Delta^{-1}=\sum_{i=0}^{d} \frac{\tau_{i}\left(\theta_{d}\right)}{\vartheta_{1} \vartheta_{2} \cdots \vartheta_{i}} \Psi^{i}
$$

provided that each of $\vartheta_{1}, \vartheta_{2}, \ldots, \vartheta_{d}$ is nonzero.

Shortly we will describe $\Psi$ and $\Delta$ in more detail, but first we restrict to the $q$-Racah case. In this case there exist nonzero scalars $a, b, q$ such that $q^{4} \neq 1$ and

$$
\theta_{i}=a q^{d-2 i}+a^{-1} q^{2 i-d}, \quad \theta_{i}^{*}=b q^{d-2 i}+b^{-1} q^{2 i-d}
$$

for $0 \leq i \leq d$. Define a linear map $K: V \rightarrow V$ such that for $0 \leq i \leq d, U_{i}$ is an eigenspace of $K$ with eigenvalue $q^{d-2 i}$. Define a linear map $B: V \rightarrow V$ such that for $0 \leq i \leq d, U_{i}^{\Downarrow}$ is an eigenspace of $B$ with eigenvalue $q^{d-2 i}$. For notational convenience define $\psi=\left(q-q^{-1}\right)\left(q^{d}-q^{-d}\right) \Psi$. By [9, Lemma 5.3] and [8, Lemma 5.4],

$$
B \Delta=\Delta K, \quad K \psi=q^{2} \psi K, \quad B \psi=q^{2} \psi B .
$$

By [8, Theorem 9.8] the map $\psi$ is equal to each of the following:

$$
\begin{array}{ll}
\frac{I-B K^{-1}}{q\left(a I-a^{-1} B K^{-1}\right)}, & \frac{I-K B^{-1}}{q\left(a^{-1} I-a K B^{-1}\right)}, \\
\frac{q\left(I-K^{-1} B\right)}{a I-a^{-1} K^{-1} B}, & \frac{q\left(I-B^{-1} K\right)}{a^{-1} I-a B^{-1} K} .
\end{array}
$$

This result is used in [8, Theorem 9.9] to obtain

$$
a K^{2}-\frac{a^{-1} q-a q^{-1}}{q-q^{-1}} K B-\frac{a q-a^{-1} q^{-1}}{q-q^{-1}} B K+a^{-1} B^{2}=0 .
$$

By (1.3) and [9, Theorem 7.2],

$$
\Delta=\exp _{q}\left(\frac{a}{q-q^{-1}} \psi\right) \exp _{q^{-1}}\left(-\frac{a^{-1}}{q-q^{-1}} \psi\right) .
$$

Motivated by this factorization, in [9, Sections 6,7] Bockting-Conrad introduces an invertible linear map $M: V \rightarrow V$ such that

$$
\begin{aligned}
& K \exp _{q}\left(\frac{a^{-1}}{q-q^{-1}} \psi\right)=\exp _{q}\left(\frac{a^{-1}}{q-q^{-1}} \psi\right) M \\
& B \exp _{q}\left(\frac{a}{q-q^{-1}} \psi\right)=\exp _{q}\left(\frac{a}{q-q^{-1}} \psi\right) M .
\end{aligned}
$$

By $[9$, Section 6],

$$
M=\frac{a K-a^{-1} B}{a-a^{-1}}, \quad M \psi=q^{2} \psi M .
$$

By [9, Lemma 6.2], $M$ is equal to each of

$$
\begin{array}{ll}
\left(I-a^{-1} q \psi\right)^{-1} K, & K\left(I-a^{-1} q^{-1} \psi\right)^{-1}, \\
(I-a q \psi)^{-1} B, & B\left(I-a q^{-1} \psi\right)^{-1} .
\end{array}
$$


By [9, Lemma 6.7],

$$
\frac{q M^{-1} K-q^{-1} K M^{-1}}{q-q^{-1}}=I, \quad \frac{q M^{-1} B-q^{-1} B M^{-1}}{q-q^{-1}}=I .
$$

We just listed many results about $\psi, \Delta, K, B, M$. In the present paper, we interpret these results in terms of polynomials. The polynomials in question are essentially (1.1), (1.2) although we adopt a more general point of view. In the next section we will describe a problem about polynomials, and for the rest of the paper we will describe the solution. In this description we will encounter analogs of the above results. We hope that the above results are illuminated by our description.

\section{Definitions and first steps}

We now begin our formal argument. The following assumptions and notational conventions apply throughout the paper. Recall the natural numbers $\mathbb{N}=\{0,1,2, \ldots\}$ and integers $\mathbb{Z}=$ $\{0, \pm 1, \pm 2, \ldots\}$. Let $\mathbb{F}$ denote an algebraically closed field. Every vector space discussed in this paper is over $\mathbb{F}$. Every algebra discussed in this paper is associative, over $\mathbb{F}$, and has a multiplicative identity. Let $x$ denote an indeterminate. Let $\mathbb{F}[x]$ denote the algebra consisting of the polynomials in $x$ that have all coefficients in $\mathbb{F}$. Throughout the paper we use the following convention: the symbols $N, n$ refer to an integer or $\infty$; the symbols $i, j, k$ refer to an integer.

We now describe a problem about polynomials. Let $N$ denote a positive integer or $\infty$. We consider an ordered pair of sequences

$$
\left\{a_{i}\right\}_{i=0}^{N-1}, \quad\left\{b_{i}\right\}_{i=0}^{N-1}
$$

such that $a_{i}, b_{i} \in \mathbb{F}$ for $0 \leq i \leq N-1$. To avoid degenerate situations, we assume that

$$
a_{0}+a_{1}+\cdots+a_{i-1} \neq b_{0}+b_{1}+\cdots+b_{i-1}, \quad 1 \leq i \leq N .
$$

We call the ordered pair (2.1) the data. For $0 \leq i \leq N$ define polynomials $\tau_{i}, \eta_{i} \in \mathbb{F}[x]$ by

$$
\begin{aligned}
\tau_{i} & =\left(x-a_{0}\right)\left(x-a_{1}\right) \cdots\left(x-a_{i-1}\right), \\
\eta_{i} & =\left(x-b_{0}\right)\left(x-b_{1}\right) \cdots\left(x-b_{i-1}\right) .
\end{aligned}
$$

The polynomials $\tau_{i}, \eta_{i}$ are monic of degree $i$. For notational convenience define $\tau_{-1}=0$ and $\eta_{-1}=0$. Let $V$ denote the subspace of $\mathbb{F}[x]$ spanned by $\left\{x^{i}\right\}_{i=0}^{N}$. Each of $\left\{\tau_{i}\right\}_{i=0}^{N},\left\{\eta_{i}\right\}_{i=0}^{N}$ is a basis for $V$. Let $\operatorname{End}(V)$ denote the algebra consisting of the $\mathbb{F}$-linear maps from $V$ to $V$. Define $\Delta \in \operatorname{End}(V)$ such that

$$
\Delta \tau_{i}=\eta_{i}, \quad 0 \leq i \leq N .
$$

Note that $\Delta$ is invertible.

Definition 2.1. An element $\psi \in \operatorname{End}(V)$ is called double lowering (with respect to the given data) whenever both

$$
\psi \tau_{i} \in \mathbb{F} \tau_{i-1}, \quad \psi \eta_{i} \in \mathbb{F} \eta_{i-1}
$$

for $0 \leq i \leq N$.

\section{Definition 2.2. Define}

$$
\mathcal{L}=\{\psi \in \operatorname{End}(V) \mid \psi \text { is double lowering }\} .
$$

Note that $\mathcal{L}$ is a subspace of the vector space $\operatorname{End}(V)$. We call $\mathcal{L}$ the double lowering space for the given data. 
Definition 2.3. The data $(2.1)$ is called double lowering whenever the double lowering space $\mathcal{L} \neq 0$.

Problem 2.4. Find necessary and sufficient conditions for the data (2.1) to be double lowering. In this case describe $\mathcal{L}$ and $\Delta$.

The above problem is solved in the present paper. The necessary and sufficient conditions are given in Theorem 12.1. By that theorem, there are four cases. For the first three cases, $\mathcal{L}$ and $\Delta$ are described in Section 6. For the fourth case, $\mathcal{L}$ and $\Delta$ are described in Section 13. We would like to acknowledge that the above problem was previously solved by R. Vidunas under the assumption that $a_{i}=\theta_{i}$ and $b_{i}=\theta_{N-i}$ for $0 \leq i \leq N-1$, with $\left\{\theta_{i}\right\}_{i=0}^{N}$ mutually distinct [41].

We have some remarks. The polynomials (2.3), (2.4) satisfy

$$
\tau_{0}=1, \quad \tau_{1}=x-a_{0}, \quad \eta_{0}=1, \quad \eta_{1}=x-b_{0} .
$$

Moreover

$$
\left(x-a_{i}\right) \tau_{i}=\tau_{i+1}, \quad\left(x-b_{i}\right) \eta_{i}=\eta_{i+1}, \quad 0 \leq i \leq N-1 .
$$

Lemma 2.5. Assume that $\psi \in \operatorname{End}(V)$ is double lowering. Then $\psi 1=0$. Moreover

$$
\psi \tau_{1}=\psi x=\psi \eta_{1},
$$

and this common value is contained in $\mathbb{F}$.

Proof. Apply $\psi$ to each side of the equations in (2.6), and use Definition 2.1.

For $0 \leq n \leq N$ define

$$
V_{n}=\operatorname{Span}\left\{x^{i}\right\}_{i=0}^{n} .
$$

We have $V_{N}=V$. We have $\operatorname{dim}\left(V_{i}\right)=i+1$ for $0 \leq i \leq N$, and $V_{i-1} \subseteq V_{i}$ for $1 \leq i \leq N$. For notational convenience define $V_{-1}=0$.

Lemma 2.6. For $0 \leq n \leq N$, each of

$$
\left\{\tau_{i}\right\}_{i=0}^{n}, \quad\left\{\eta_{i}\right\}_{i=0}^{n}
$$

is a basis for $V_{n}$.

Proof. Each of $\tau_{i}, \eta_{i}$ has degree $i$ for $0 \leq i \leq N$.

Lemma 2.7. For $\psi \in \mathcal{L}$ and $0 \leq i \leq N$,

$$
\psi V_{i} \subseteq V_{i-1} .
$$

Moreover $\psi^{i+1} V_{i}=0$.

Proof. By Definition 2.1 and Lemma 2.6.

For $T \in \operatorname{End}(V), T$ is called nilpotent whenever there exists a positive integer $j$ such that $T^{j}=0$. The map $T$ is called locally nilpotent whenever for all $v \in V$ there exists a positive integer $j$ such that $T^{j} v=0$. If $T$ is nilpotent then $T$ is locally nilpotent. For $N \neq \infty$, if $T$ is locally nilpotent then $T$ is nilpotent.

Lemma 2.8. Each element of $\mathcal{L}$ is locally nilpotent. If $N \neq \infty$ then each element of $\mathcal{L}$ is nilpotent. 
Proof. By Lemma 2.7 and the comments below it.

Lemma 2.9. For $1 \leq i \leq N$,

(i) in $\tau_{i}$ the coefficient of $x^{i-1}$ is

$$
-a_{0}-a_{1}-\cdots-a_{i-1}
$$

(ii) in $\eta_{i}$ the coefficient of $x^{i-1}$ is

$$
-b_{0}-b_{1}-\cdots-b_{i-1} \text {. }
$$

Proof. Use (2.3), (2.4).

Lemma 2.10. For $1 \leq i \leq N$,

(i) $\eta_{i}-\tau_{i} \in V_{i-1}$;

(ii) in $\eta_{i}-\tau_{i}$ the coefficient of $x^{i-1}$ is

$$
a_{0}+a_{1}+\cdots+a_{i-1}-b_{0}-b_{1}-\cdots-b_{i-1} .
$$

Proof. (i) Each of $\tau_{i}, \eta_{i}$ is monic with degree $i$. (ii) Use Lemma 2.9.

Definition 2.11. Define a map $A: V_{N-1} \rightarrow V_{N}, v \mapsto x v$. Note that $A$ is $\mathbb{F}$-linear.

Lemma 2.12. We have

$$
A V_{n-1} \subseteq V_{n}, \quad 1 \leq n \leq N .
$$

Proof. By (2.9).

Lemma 2.13. For $0 \leq i \leq N-1$,

$$
A \tau_{i}=a_{i} \tau_{i}+\tau_{i+1}, \quad A \eta_{i}=b_{i} \eta_{i}+\eta_{i+1} .
$$

Proof. By (2.7) and Definition 2.11.

We mention an elementary result for later use.

Lemma 2.14. Assume that $T \in \operatorname{End}(V)$ is locally nilpotent. Then $I-T$ is invertible, and $(I-T)^{-1}=\sum_{i=0}^{N} T^{i}$.

\section{Adjusting the data}

In this section we describe how the double lowering space is affected when we adjust the data in an affine way.

Let $\mathrm{GL}_{2}(\mathbb{F})$ denote the group of invertible $2 \times 2$ matrices that have all entries in $\mathbb{F}$.

Definition 3.1. Let $G$ denote the subgroup of $\mathrm{GL}_{2}(\mathbb{F})$ consisting of the matrices

$$
\left(\begin{array}{ll}
1 & t \\
0 & s
\end{array}\right), \quad 0 \neq s \in \mathbb{F}, \quad t \in \mathbb{F}
$$

The above matrix is denoted $g(s, t)$.

Lemma 3.2. With reference to Definition 3.1, 
(i) $g(s, t) g(S, T)=g(s S, T+t S)$;

(ii) the inverse of $g(s, t)$ is $g\left(s^{-1},-s^{-1} t\right)$.

Proof. Routine matrix multiplication.

For an algebra $\mathcal{A}$, an automorphism of $\mathcal{A}$ is an algebra isomorphism $\mathcal{A} \rightarrow \mathcal{A}$.

Lemma 3.3. The group $G$ acts on the algebra $\mathbb{F}[x]$ as a group of automorphisms in the following way: each element $g(s, t) \in G$ sends $x \mapsto s x+t$.

Proof. This is routinely checked using Lemma 3.2.

Recall from Definition 2.2 the double lowering space $\mathcal{L}$ for the data $(2.1)$. Pick $0 \neq s \in \mathbb{F}$ and $t \in \mathbb{F}$. Let $\mathcal{L}^{\prime}$ denote the double lowering space for the data

$$
\left\{s a_{i}+t\right\}_{i=0}^{N-1}, \quad\left\{s b_{i}+t\right\}_{i=0}^{N-1} .
$$

Proposition 3.4. The following $(i)$-(iii) hold for the above scalars $s, t$ and $g=g(s, t)$ :

(i) there exists an $\mathbb{F}$-linear map $\mathcal{L} \rightarrow \mathcal{L}^{\prime}, \psi \mapsto g^{-1} \psi g$;

(ii) there exists an $\mathbb{F}$-linear map $\mathcal{L}^{\prime} \rightarrow \mathcal{L}, \zeta \mapsto g \zeta g^{-1}$;

(iii) the maps in (i), (ii) above are inverses, and hence bijections.

Proof. (i) For $\psi \in \mathcal{L}$ we show that $g^{-1} \psi g \in \mathcal{L}^{\prime}$. For $\alpha \in \mathbb{F}$ define $\alpha^{\prime}=s \alpha+t$. For $0 \leq i \leq N$ define

$$
\begin{aligned}
\tau_{i}^{\prime} & =\left(x-a_{0}^{\prime}\right)\left(x-a_{1}^{\prime}\right) \cdots\left(x-a_{i-1}^{\prime}\right), \\
\eta_{i}^{\prime} & =\left(x-b_{0}^{\prime}\right)\left(x-b_{1}^{\prime}\right) \cdots\left(x-b_{i-1}^{\prime}\right) .
\end{aligned}
$$

For notational convenience define $\tau_{-1}^{\prime}=0$ and $\eta_{-1}^{\prime}=0$. Pick an integer $i, 0 \leq i \leq N$. One checks that $g$ sends

$$
\tau_{i}^{\prime} \mapsto s^{i} \tau_{i}, \quad \eta_{i}^{\prime} \mapsto s^{i} \eta_{i}
$$

By Definition 2.1, $\psi$ sends $\tau_{i}$ (resp. $\eta_{i}$ ) to a scalar multiple of $\tau_{i-1}$ (resp. $\eta_{i-1}$ ). By these comments $g^{-1} \psi g$ sends $\tau_{i}^{\prime}$ (resp. $\eta_{i}^{\prime}$ ) to a scalar multiple of $\tau_{i-1}^{\prime}$ (resp. $\eta_{i-1}^{\prime}$ ). Therefore $g^{-1} \psi g \in \mathcal{L}^{\prime}$. We have shown that there exists a map $\mathcal{L} \rightarrow \mathcal{L}^{\prime}, \psi \mapsto g^{-1} \psi g$. By construction this map is F-linear.

(ii) Similar to the proof of (i) above. (iii) By construction.

Corollary 3.5. Pick $0 \neq s \in \mathbb{F}$ and $t \in \mathbb{F}$. Then the data (2.1) is double lowering if and only if the data

$$
\left\{s a_{i}+t\right\}_{i=0}^{N-1}, \quad\left\{s b_{i}+t\right\}_{i=0}^{N-1}
$$

is double lowering.

Proof. By Definition 2.3 and Proposition 3.4.

We have a comment.

Lemma 3.6. Referring to the data (2.1), for distinct $a_{0}^{\prime}, b_{0}^{\prime} \in \mathbb{F}$ define

$$
s=\frac{a_{0}^{\prime}-b_{0}^{\prime}}{a_{0}-b_{0}}, \quad t=\frac{a_{0} b_{0}^{\prime}-a_{0}^{\prime} b_{0}}{a_{0}-b_{0}} .
$$

Then $s \neq 0$ and

$$
s a_{0}+t=a_{0}^{\prime}, \quad s b_{0}+t=b_{0}^{\prime} .
$$

Proof. Routine.

Corollary 3.5 and Lemma 3.6 show that for double lowering data (2.1), the scalars $a_{0}$ and $b_{0}$ are "free", with $a_{0} \neq b_{0}$ being the only constraint. 


\section{The parameters $\vartheta_{i}$}

We continue to discuss the double lowering space $\mathcal{L}$ for the data $(2.1)$. In this section we use $\mathcal{L}$ to define some scalars $\left\{\vartheta_{i}\right\}_{i=0}^{N}$ that will play a role in our theory.

Definition 4.1. For $0 \leq i \leq N$ define

$$
\vartheta_{i}=\frac{a_{0}+a_{1}+\cdots+a_{i-1}-b_{0}-b_{1}-\cdots-b_{i-1}}{a_{0}-b_{0}} .
$$

Referring to Definition 4.1, we have $\vartheta_{0}=0$ and $\vartheta_{1}=1$. By $(2.2)$ we have

$$
\vartheta_{i} \neq 0, \quad 1 \leq i \leq N .
$$

Proposition 4.2. The following $(i)-($ iii) hold for $\psi \in \mathcal{L}$ and $1 \leq i \leq N$ :

(i) for the polynomial $\psi x^{i}$ the coefficient of $x^{i-1}$ is $\vartheta_{i} \psi x$;

(ii) $\psi \tau_{i}=\left(\vartheta_{i} \psi x\right) \tau_{i-1}$;

(iii) $\psi \eta_{i}=\left(\vartheta_{i} \psi x\right) \eta_{i-1}$.

Proof. We use induction on $i$. First assume that $i=1$, and recall $\vartheta_{1}=1$. Assertion (i) is vacuously true. Assertions (ii), (iii) hold by (2.8) and since $\tau_{0}=1=\eta_{0}$. Next assume that $i \geq 2$. By Lemma 2.7, $\psi x^{i} \in V_{i-1}$. Let the scalar $\alpha_{i}$ be the coefficient of $x^{i-1}$ in $\psi x^{i}$. Since $\psi \in \mathcal{L}, \psi \tau_{i} \in \mathbb{F} \tau_{i-1}$. Since each of $\tau_{i}, \tau_{i-1}$ is monic,

$$
\psi \tau_{i}=\alpha_{i} \tau_{i-1} .
$$

Similarly,

$$
\psi \eta_{i}=\alpha_{i} \eta_{i-1}
$$

We show that $\alpha_{i}=\vartheta_{i} \psi x$. Using (4.1), (4.2) we see that

$$
\left(a_{0}-b_{0}\right) \vartheta_{i-1} x^{i-2}
$$

times

$$
\alpha_{i}-\vartheta_{i} \psi x
$$

is equal to

$$
\psi\left(\eta_{i}-\tau_{i}\right)-\left(a_{0}-b_{0}\right) \vartheta_{i} \psi x^{i-1}
$$

minus $\alpha_{i}$ times

$$
\eta_{i-1}-\tau_{i-1}-\left(a_{0}-b_{0}\right) \vartheta_{i-1} x^{i-2}
$$

plus $\left(a_{0}-b_{0}\right) \vartheta_{i}$ times

$$
\psi x^{i-1}-(\psi x) \vartheta_{i-1} x^{i-2} .
$$

By Lemma 2.10 and Definition 4.1,

$$
\eta_{i}-\tau_{i}-\left(a_{0}-b_{0}\right) \vartheta_{i} x^{i-1} \in V_{i-2} .
$$

In this inclusion, we apply $\psi$ to each side and use Lemma 2.7 to find that (4.5) is contained in $V_{i-3}$. In (4.8) we replace $i$ by $i-1$, to find that (4.6) is contained in $V_{i-3}$. By induction (4.7) is contained in $V_{i-3}$. By these comments the polynomial (4.3) times the scalar (4.4) is contained in $V_{i-3}$. Consider the factors in the polynomial (4.3). We have $a_{0}-b_{0} \neq 0$ and $\vartheta_{i-1} \neq 0$ and $x^{i-2} \notin V_{i-3}$. So the polynomial (4.3) is not contained in $V_{i-3}$. Consequently the scalar (4.4) is zero, so $\alpha_{i}=\vartheta_{i} \psi x$. The result follows from this and (4.1), (4.2). 
Corollary 4.3. The map $\mathcal{L} \rightarrow \mathbb{F}, \psi \mapsto \psi x$ is injective.

Proof. For $\psi \in \mathcal{L}$ such that $\psi x=0$, we show that $\psi=0$. The vector space $V$ has a basis $\left\{\tau_{i}\right\}_{i=0}^{N}$. By Definition 2.1, $\psi \tau_{0}=0$. By Proposition 4.2(ii), $\psi \tau_{i}=0$ for $1 \leq i \leq N$. By these comments $\psi=0$.

Corollary 4.4. Assume that $\mathcal{L} \neq 0$. Then the map in Corollary 4.3 is a bijection. Moreover $\mathcal{L}$ has dimension 1.

Proof. By Corollary 4.3.

Definition 4.5. An element $\psi \in \mathcal{L}$ is called normalized whenever $\psi x=1$.

Lemma 4.6. The double lowering space $\mathcal{L}$ contains a normalized element if and only if $\mathcal{L} \neq 0$; in this case the normalized element is unique.

Proof. By Corollary 4.4 and Definition 4.5.

Lemma 4.7. Assume that $\mathcal{L} \neq 0$. Then the vector space $\mathcal{L}$ has a basis consisting of its normalized element.

Proof. The vector space $\mathcal{L}$ has dimension 1, and its normalized element is nonzero.

Lemma 4.8. For $\psi \in \operatorname{End}(V)$ the following are equivalent:

(i) $\psi \in \mathcal{L}$ and $\psi$ is normalized;

(ii) for $0 \leq i \leq N$ both

$$
\psi \tau_{i}=\vartheta_{i} \tau_{i-1}, \quad \psi \eta_{i}=\vartheta_{i} \eta_{i-1}
$$

Proof. (i) $\Rightarrow$ (ii) Set $\psi x=1$ in Proposition $4.2($ ii), (iii). (ii) $\Rightarrow$ (i) We have $\psi \in \mathcal{L}$ by Definition 2.2 and (4.9). To see that $\psi$ is normalized, set $i=1$ in (4.9) and use Lemma 2.5 to obtain $\psi x=1$.

\section{$5 \quad$ Describing $\mathcal{L}$ using $\Delta$}

We continue to discuss the double lowering space $\mathcal{L}$ for the data (2.1). In this section we describe $\mathcal{L}$ using the map $\Delta$ from (2.5).

Proposition 5.1. For $\psi \in \operatorname{End}(V)$ the following $(i)-(i i i)$ are equivalent:

(i) $\Delta \psi=\psi \Delta$ and

$$
\psi \tau_{i} \in \mathbb{F} \tau_{i-1}, \quad 0 \leq i \leq N
$$

(ii) $\Delta \psi=\psi \Delta$ and

$$
\psi \eta_{i} \in \mathbb{F} \eta_{i-1}, \quad 0 \leq i \leq N
$$

(iii) $\psi \in \mathcal{L}$. 
Proof. (i) $\Rightarrow$ (ii) We show (5.2). Using (2.5),

$$
\psi \eta_{i}=\psi \Delta \tau_{i}=\Delta \psi \tau_{i} \in \mathbb{F} \Delta \tau_{i-1}=\mathbb{F} \eta_{i-1} .
$$

(ii) $\Rightarrow$ (i) Similar to the proof of (i) $\Rightarrow$ (ii). (i), (ii) $\Rightarrow$ (iii) By Definitions 2.1, 2.2. (iii) $\Rightarrow$ (i) We have (5.1) by Definition 2.2. We show $\Delta \psi=\psi \Delta$. By Lemma 4.7 we may assume that $\psi$ is normalized. The vector space $V$ has a basis $\left\{\tau_{i}\right\}_{i=0}^{N}$. By Lemma 4.8 we have

$$
\psi \tau_{i}=\vartheta_{i} \tau_{i-1}, \quad \psi \eta_{i}=\vartheta_{i} \eta_{i-1}, \quad 0 \leq i \leq N .
$$

So for $0 \leq i \leq N$,

$$
\Delta \psi \tau_{i}=\vartheta_{i} \Delta \tau_{i-1}=\vartheta_{i} \eta_{i-1}=\psi \eta_{i}=\psi \Delta \tau_{i} .
$$

By these comments $\Delta \psi=\psi \Delta$.

We introduce some notation. For $0 \leq i \leq j \leq N$ define

$$
\left[\begin{array}{l}
j \\
i
\end{array}\right]_{\vartheta}=\frac{\vartheta_{j} \vartheta_{j-1} \cdots \vartheta_{j-i+1}}{\vartheta_{1} \vartheta_{2} \cdots \vartheta_{i}}
$$

Note that

$$
\left[\begin{array}{l}
j \\
i
\end{array}\right]_{\vartheta}=\left[\begin{array}{c}
j \\
j-i
\end{array}\right]_{\vartheta}, \quad 0 \leq i \leq j \leq N .
$$

Proposition 5.2. The following $(i)-($ iii) are equivalent:

(i) $\mathcal{L} \neq 0$;

(ii) for $0 \leq j \leq N$,

$$
\eta_{j}=\sum_{i=0}^{j} \eta_{j-i}\left(a_{0}\right)\left[\begin{array}{l}
j \\
i
\end{array}\right]_{\vartheta} \tau_{i} ;
$$

(iii) for $0 \leq j \leq N$,

$$
\tau_{j}=\sum_{i=0}^{j} \tau_{j-i}\left(b_{0}\right)\left[\begin{array}{l}
j \\
i
\end{array}\right]_{\vartheta} \eta_{i} .
$$

Assume that $(i)-($ iii $)$ hold, and let $\psi \in \mathcal{L}$ be normalized. Then

$$
\begin{aligned}
& \Delta=\sum_{i=0}^{N} \frac{\eta_{i}\left(a_{0}\right)}{\vartheta_{1} \vartheta_{2} \cdots \vartheta_{i}} \psi^{i}, \\
& \Delta^{-1}=\sum_{i=0}^{N} \frac{\tau_{i}\left(b_{0}\right)}{\vartheta_{1} \vartheta_{2} \cdots \vartheta_{i}} \psi^{i} .
\end{aligned}
$$

Proof. (i) $\Rightarrow$ (ii) Let $j$ be given. There exist scalars $\left\{\alpha_{i}\right\}_{i=0}^{j}$ in $\mathbb{F}$ such that

$$
\eta_{j}=\sum_{i=0}^{j} \alpha_{i} \tau_{i} .
$$


For $0 \leq i \leq j$ we show

$$
\alpha_{i}=\eta_{j-i}\left(a_{0}\right)\left[\begin{array}{l}
j \\
i
\end{array}\right]_{\vartheta}
$$

Let $\psi \in \mathcal{L}$ be normalized. In (5.7) we apply $\psi^{i}$ to each side, and evaluate the result using Lemma 4.8. We then set $x=a_{0}$, and use the fact that $\tau_{0}=1$ and $\tau_{k}\left(a_{0}\right)=0$ for $1 \leq k \leq N$. By these comments

$$
\eta_{j-i}\left(a_{0}\right) \vartheta_{j} \vartheta_{j-1} \cdots \vartheta_{j-i+1}=\alpha_{i} \vartheta_{1} \vartheta_{2} \cdots \vartheta_{i}
$$

From this equation we obtain (5.8). By (5.7), (5.8) we obtain (5.4), so (ii) holds.

(ii) $\Rightarrow$ (i) Define $\psi \in \operatorname{End}(V)$ such that $\psi \tau_{i}=\vartheta_{i} \tau_{i-1}$ for $0 \leq i \leq N$. We have $\psi \neq 0$ since $N \geq 1$ and $\vartheta_{1}=1, \tau_{0}=1$. We show $\psi \in \mathcal{L}$. To do this, it is convenient to first show that $\psi$ satisfies (5.5). To obtain (5.5), for $0 \leq j \leq N$ we apply each side of (5.5) to $\tau_{j}$. Concerning the left-hand side of (5.5), we have $\Delta \tau_{j}=\eta_{j}$ by (2.5). Concerning the right-hand side of (5.5),

$$
\begin{aligned}
\sum_{i=0}^{j} \frac{\eta_{i}\left(a_{0}\right)}{\vartheta_{1} \vartheta_{2} \cdots \vartheta_{i}} \psi^{i} \tau_{j} & =\sum_{i=0}^{j} \frac{\eta_{i}\left(a_{0}\right) \vartheta_{j} \vartheta_{j-1} \cdots \vartheta_{j-i+1}}{\vartheta_{1} \vartheta_{2} \cdots \vartheta_{i}} \tau_{j-i}=\sum_{i=0}^{j} \eta_{i}\left(a_{0}\right)\left[\begin{array}{c}
j \\
i
\end{array}\right]_{\vartheta} \tau_{j-i} \\
& =\sum_{i=0}^{j} \eta_{j-i}\left(a_{0}\right)\left[\begin{array}{c}
j \\
i
\end{array}\right]_{\vartheta} \tau_{i}=\eta_{j} .
\end{aligned}
$$

We have shown that each side of (5.5) sends $\tau_{j} \mapsto \eta_{j}$ for $0 \leq j \leq N$. Therefore (5.5) holds. By (5.5), $\Delta$ is a polynomial in $\psi$. Consequently $\psi \Delta=\Delta \psi$. The map $\psi$ satisfies Proposition 5.1(i), so $\psi \in \mathcal{L}$ by Proposition 5.1(i), (iii). Therefore $\mathcal{L} \neq 0$.

(i) $\Leftrightarrow$ (iii) Interchange the roles of $\left\{a_{i}\right\}_{i=0}^{N-1},\left\{b_{i}\right\}_{i=0}^{N-1}$ in the proof of (i) $\Leftrightarrow$ (ii).

Now assume that (i)-(iii) hold. We saw in the proof of (ii) $\Rightarrow$ (i) that (5.5) holds. Interchanging the roles of $\left\{a_{i}\right\}_{i=0}^{N-1},\left\{b_{i}\right\}_{i=0}^{N-1}$ in that proof, we see that (5.6) holds.

Later in the paper, we will obtain necessary and sufficient conditions for the data (2.1) to satisfy conditions (i)-(iii) in Proposition 5.2; our result is Theorem 12.1. In order to motivate this result, we look at some examples of double lowering data. This will be done in the next section.

\section{First examples of double lowering data}

We continue to discuss the double lowering space $\mathcal{L}$ for the data (2.1). In this section we give three assumptions under which this data is double lowering. Under each assumption we describe the polynomials $\left\{\tau_{i}\right\}_{i=0}^{N-1},\left\{\eta_{i}\right\}_{i=0}^{N-1}$ from (2.3), (2.4), the parameters $\left\{\vartheta_{i}\right\}_{i=0}^{N}$ from Definition 4.1, and the map $\Delta$ from (2.5).

As a warmup, we examine the condition (5.4) for some small values of $j$.

Lemma 6.1. The following $(i)-(i v)$ hold.

(i) $\eta_{0}=\tau_{0}$

(ii) $\eta_{1}=\eta_{1}\left(a_{0}\right) \tau_{0}+\tau_{1}$.

(iii) For $N \geq 2$,

$$
\eta_{2}=\eta_{2}\left(a_{0}\right) \tau_{0}+\vartheta_{2} \eta_{1}\left(a_{0}\right) \tau_{1}+\tau_{2}
$$


(iv) For $N \geq 3$,

$$
\eta_{3}=\eta_{3}\left(a_{0}\right) \tau_{0}+\vartheta_{3} \eta_{2}\left(a_{0}\right) \tau_{1}+\vartheta_{3} \eta_{1}\left(a_{0}\right) \tau_{2}+\tau_{3}+\left(x-a_{0}\right) \varepsilon
$$

where

$$
\varepsilon=\left(b_{0}-a_{1}\right)\left(b_{2}-a_{1}\right)-\left(a_{0}-b_{1}\right)\left(a_{2}-b_{1}\right) .
$$

Proof. To verify these equations, evaluate the terms using (2.3), (2.4) and Definition 4.1.

Lemma 6.2. Assume that $N \leq 2$. Then $\mathcal{L} \neq 0$.

Proof. By Proposition 5.2(i), (ii) and Lemma 6.1.

Lemma 6.3. Assume that $N=3$. Then $\mathcal{L} \neq 0$ if and only if

$$
\left(a_{0}-b_{1}\right)\left(a_{2}-b_{1}\right)=\left(b_{0}-a_{1}\right)\left(b_{2}-a_{1}\right) \text {. }
$$

Proof. By Proposition 5.2(i),(ii) and Lemma 6.1.

Lemma 6.4. Assume that $a_{i-1}=b_{i}$ for $1 \leq i \leq N-1$. Then the following (i)-(v) hold:

(i) $\mathcal{L} \neq 0$;

(ii) $\eta_{i}=\left(x-b_{0}\right) \tau_{i-1}$ for $1 \leq i \leq N$;

(iii) $\eta_{i}\left(a_{0}\right)=0$ for $2 \leq i \leq N$;

(iv) $\vartheta_{i}=\frac{a_{i-1}-b_{0}}{a_{0}-b_{0}}$ for $1 \leq i \leq N$;

(v) $\Delta=I+\left(a_{0}-b_{0}\right) \psi$, where $\psi \in \mathcal{L}$ is normalized.

Proof. (ii) By (2.3), (2.4). (iii) By (ii) and since $\tau_{j}\left(a_{0}\right)=0$ for $1 \leq j \leq N$. (iv) Use Definition 4.1. (i) Apply Proposition 5.2(i), (ii). (v) By (5.5) and (iii) above.

Lemma 6.5. Assume that $a_{i}=b_{i-1}$ for $1 \leq i \leq N-1$. Then the following $(i)-(v)$ hold:

(i) $\mathcal{L} \neq 0$;

(ii) $\tau_{i}=\left(x-a_{0}\right) \eta_{i-1}$ for $1 \leq i \leq N$;

(iii) $\tau_{i}\left(b_{0}\right)=0$ for $2 \leq i \leq N$;

(iv) $\vartheta_{i}=\frac{a_{0}-b_{i-1}}{a_{0}-b_{0}}$ for $1 \leq i \leq N$;

(v) $\Delta^{-1}=I+\left(b_{0}-a_{0}\right) \psi$, where $\psi \in \mathcal{L}$ is normalized.

Proof. Interchange the roles of $\left\{a_{i}\right\}_{i=0}^{N-1},\left\{b_{i}\right\}_{i=0}^{N-1}$ in Lemma 6.4.

For the rest of this section, assume that $N \geq 2$. Also for the rest of this section, fix $\theta \in \mathbb{F}$ and assume

$$
\begin{aligned}
& a_{0} \neq \theta, \quad b_{0} \neq \theta ; \\
& a_{i}=\theta, \quad b_{i}=\theta, \quad 1 \leq i \leq N-2 ; \\
& \frac{\theta-a_{N-1}}{\theta-b_{0}}=\frac{\theta-b_{N-1}}{\theta-a_{0}} \quad \text { if } N \neq \infty .
\end{aligned}
$$

Using Definition 4.1,

$$
\vartheta_{i}=1, \quad 1 \leq i \leq N-1
$$


and for $N \neq \infty$,

$$
a_{N-1}=b_{0}+\vartheta_{N}\left(\theta-b_{0}\right), \quad b_{N-1}=a_{0}+\vartheta_{N}\left(\theta-a_{0}\right) .
$$

Using (5.3),

$$
\left[\begin{array}{l}
j \\
i
\end{array}\right]_{\vartheta}=1, \quad 0 \leq i \leq j \leq N-1
$$

and for $N \neq \infty$,

$$
\left[\begin{array}{c}
N \\
i
\end{array}\right]_{\vartheta}=\vartheta_{N}, \quad 1 \leq i \leq N-1 .
$$

For $0 \leq i \leq N$ the polynomials $\tau_{i}, \eta_{i}$ are described in the table below:

\begin{tabular}{c|cc}
$i$ & $\tau_{i}$ & $\eta_{i}$ \\
\hline 0 & 1 & 1 \\
$1 \leq i \leq N-1$ & $\left(x-a_{0}\right)(x-\theta)^{i-1}$ & $\left(x-b_{0}\right)(x-\theta)^{i-1}$ \\
$N$ & $\left(x-a_{0}\right)(x-\theta)^{N-2}\left(x-a_{N-1}\right)$ & $\left(x-b_{0}\right)(x-\theta)^{N-2}\left(x-b_{N-1}\right)$
\end{tabular}

For $1 \leq i \leq N$ the values of $\eta_{i}-\tau_{i}$ and $\eta_{i}\left(a_{0}\right)$ are described in the table below:

\begin{tabular}{c|cc}
$i$ & $\eta_{i}-\tau_{i}$ & $\eta_{i}\left(a_{0}\right)$ \\
\hline $1 \leq i \leq N-1$ & $\left(a_{0}-b_{0}\right)(x-\theta)^{i-1}$ & $\left(a_{0}-b_{0}\right)\left(a_{0}-\theta\right)^{i-1}$ \\
$N$ & $\vartheta_{N}\left(a_{0}-b_{0}\right)(x-\theta)^{N-1}$ & $\vartheta_{N}\left(a_{0}-b_{0}\right)\left(a_{0}-\theta\right)^{N-1}$
\end{tabular}

Lemma 6.6. Under assumptions (6.1)-(6.3) the following (i)-(iii) hold:

(i) $\mathcal{L} \neq 0$;

(ii) $\Delta=\frac{I+\left(\theta-b_{0}\right) \psi}{I+\left(\theta-a_{0}\right) \psi}$;

(iii) $\Delta^{-1}=\frac{I+\left(\theta-a_{0}\right) \psi}{I+\left(\theta-b_{0}\right) \psi}$.

In the above lines $\psi \in \mathcal{L}$ is normalized.

Proof. (i) We invoke Proposition 5.2(i), (ii). For $0 \leq j \leq N$ we verify (5.4). We may assume that $2 \leq j \leq N$; otherwise we are done by Lemma 6.1. For $N \neq \infty$ we separate the cases $2 \leq j \leq N-1$ and $j=N$. It suffices to show that

$$
\begin{aligned}
& \eta_{j}=\tau_{j}+\eta_{j}\left(a_{0}\right)+\sum_{i=1}^{j-1} \eta_{j-i}\left(a_{0}\right) \tau_{i}, \quad 2 \leq j \leq N-1, \\
& \eta_{N}=\tau_{N}+\eta_{N}\left(a_{0}\right)+\vartheta_{N} \sum_{i=1}^{N-1} \eta_{N-i}\left(a_{0}\right) \tau_{i}, \quad \text { if } N \neq \infty .
\end{aligned}
$$

For $2 \leq j \leq N$ the values of $\eta_{j}-\tau_{j}$ and $\eta_{j}\left(a_{0}\right)$ are given in the table above the lemma statement. Also for $2 \leq j \leq N$,

$$
\begin{aligned}
\sum_{i=1}^{j-1} \eta_{j-i}\left(a_{0}\right) \tau_{i} & =\left(a_{0}-b_{0}\right)\left(x-a_{0}\right) \sum_{i=1}^{j-1}\left(a_{0}-\theta\right)^{j-i-1}(x-\theta)^{i-1} \\
& =\left(a_{0}-b_{0}\right)\left(x-a_{0}\right)\left(a_{0}-\theta\right)^{j-2} \sum_{k=0}^{j-2}\left(\frac{x-\theta}{a_{0}-\theta}\right)^{k} \\
& =\left(a_{0}-b_{0}\right)(x-\theta)^{j-1}-\left(a_{0}-b_{0}\right)\left(a_{0}-\theta\right)^{j-1} .
\end{aligned}
$$

Using the above comments we routinely verify $(6.4),(6.5)$. 
(ii) We will verify the equation by showing that the two sides agree on $V_{j}$ for $2 \leq j \leq N$. Using (5.5) and $\psi^{j+1} V_{j}=0$ we see that on $V_{j}$,

$$
\begin{aligned}
\Delta-I & =\sum_{i=1}^{j} \frac{\eta_{i}\left(a_{0}\right)}{\vartheta_{1} \vartheta_{2} \cdots \vartheta_{i}} \psi^{i}=\frac{\eta_{j}\left(a_{0}\right)}{\vartheta_{j}} \psi^{j}+\sum_{i=1}^{j-1} \eta_{i}\left(a_{0}\right) \psi^{i}=\left(a_{0}-b_{0}\right) \psi \sum_{k=0}^{j-1}\left(a_{0}-\theta\right)^{k} \psi^{k} \\
& =\left(a_{0}-b_{0}\right) \psi \frac{I-\left(a_{0}-\theta\right)^{j} \psi^{j}}{I-\left(a_{0}-\theta\right) \psi}=\frac{\left(a_{0}-b_{0}\right) \psi}{I-\left(a_{0}-\theta\right) \psi} .
\end{aligned}
$$

The result follows. (iii) By (ii) above.

We just gave some examples of double lowering data. There is another example that is somewhat more involved; it will be described later in the paper.

\section{Extending the data}

Throughout this section, we assume that $N$ is an integer at least 2. Recall the data $\left\{a_{i}\right\}_{i=0}^{N-1}$, $\left\{b_{i}\right\}_{i=0}^{N-1}$ from (2.1), and assume that this data is double lowering. Let $a_{N}, b_{N} \in \mathbb{F}$ satisfy

$$
a_{0}+a_{1}+\cdots+a_{N} \neq b_{0}+b_{1}+\cdots+b_{N}
$$

giving data

$$
\left\{a_{i}\right\}_{i=0}^{N}, \quad\left\{b_{i}\right\}_{i=0}^{N} .
$$

In this section we obtain necessary and sufficient conditions on $a_{N}, b_{N}$ for the data (7.1) to be double lowering. By (2.7),

$$
\tau_{N+1}=\left(x-a_{N}\right) \tau_{N}, \quad \eta_{N+1}=\left(x-b_{N}\right) \eta_{N} .
$$

Lemma 7.1. The following $(i)-($ iii $)$ are equivalent:

(i) the data (7.1) is double lowering;

(ii) we have

$$
\eta_{N+1}=\sum_{i=0}^{N+1} \eta_{N-i+1}\left(a_{0}\right)\left[\begin{array}{c}
N+1 \\
i
\end{array}\right]_{\vartheta} \tau_{i}
$$

(iii) we have

$$
\tau_{N+1}=\sum_{i=0}^{N+1} \tau_{N-i+1}\left(b_{0}\right)\left[\begin{array}{c}
N+1 \\
i
\end{array}\right]_{\vartheta} \eta_{i} .
$$

Proof. By Proposition 5.2.

Lemma 7.2. We have

$$
\begin{aligned}
\eta_{N+1} & =\sum_{i=0}^{N} \eta_{N-i}\left(a_{0}\right)\left[\begin{array}{c}
N \\
i
\end{array}\right]_{\vartheta}\left(a_{i}-b_{N}\right) \tau_{i}+\sum_{i=1}^{N+1} \eta_{N-i+1}\left(a_{0}\right)\left[\begin{array}{c}
N \\
i-1
\end{array}\right]_{\vartheta} \tau_{i}, \\
\tau_{N+1} & =\sum_{i=0}^{N} \tau_{N-i}\left(b_{0}\right)\left[\begin{array}{c}
N \\
i
\end{array}\right]_{\vartheta}\left(b_{i}-a_{N}\right) \eta_{i}+\sum_{i=1}^{N+1} \tau_{N-i+1}\left(b_{0}\right)\left[\begin{array}{c}
N \\
i-1
\end{array}\right]_{\vartheta} \eta_{i} .
\end{aligned}
$$


Proof. We show (7.3). Using Proposition 5.2,

$$
\begin{aligned}
\eta_{N+1} & =\left(x-b_{N}\right) \eta_{N}=\left(x-b_{N}\right) \sum_{i=0}^{N} \eta_{N-i}\left(a_{0}\right)\left[\begin{array}{c}
N \\
i
\end{array}\right]_{\vartheta} \tau_{i} \\
& =\sum_{i=0}^{N} \eta_{N-i}\left(a_{0}\right)\left[\begin{array}{c}
N \\
i
\end{array}\right]_{\vartheta}\left(\left(a_{i}-b_{N}\right) \tau_{i}+\tau_{i+1}\right) \\
& =\sum_{i=0}^{N} \eta_{N-i}\left(a_{0}\right)\left[\begin{array}{c}
N \\
i
\end{array}\right]_{\vartheta}\left(a_{i}-b_{N}\right) \tau_{i}+\sum_{i=1}^{N+1} \eta_{N-i+1}\left(a_{0}\right)\left[\begin{array}{c}
N \\
i-1
\end{array}\right]_{\vartheta} \tau_{i} .
\end{aligned}
$$

Line (7.4) is similarly obtained.

Proposition 7.3. The following $(i)-($ iii) are equivalent:

(i) the data (7.1) is double lowering;

(ii) for $0 \leq i \leq N-1$ such that $\eta_{i}\left(a_{0}\right) \neq 0$,

$$
\begin{aligned}
& \left(a_{0}+\cdots+a_{i}-b_{0}-\cdots-b_{i}\right)\left(a_{N-i}-b_{N}\right) \\
& \quad=\left(a_{0}-b_{i}\right)\left(a_{N-i}+\cdots+a_{N}-b_{N-i}-\cdots-b_{N}\right) ;
\end{aligned}
$$

(iii) for $0 \leq i \leq N-1$ such that $\tau_{i}\left(b_{0}\right) \neq 0$,

$$
\begin{aligned}
& \left(b_{0}+\cdots+b_{i}-a_{0}-\cdots-a_{i}\right)\left(b_{N-i}-a_{N}\right) \\
& \quad=\left(b_{0}-a_{i}\right)\left(b_{N-i}+\cdots+b_{N}-a_{N-i}-\cdots-a_{N}\right) .
\end{aligned}
$$

Proof. (i) $\Leftrightarrow($ ii) We invoke Lemma 7.1(i), (ii). Subtract (7.2) from (7.3) to obtain an equation $0=\sum_{i=1}^{N} d_{i} \tau_{i}$ where

$$
d_{i}=\eta_{N-i}\left(a_{0}\right)\left[\begin{array}{c}
N \\
i
\end{array}\right]_{\vartheta}\left(a_{i}-b_{N}\right)+\eta_{N-i+1}\left(a_{0}\right)\left[\begin{array}{c}
N \\
i-1
\end{array}\right]_{\vartheta}-\eta_{N-i+1}\left(a_{0}\right)\left[\begin{array}{c}
N+1 \\
i
\end{array}\right]_{\vartheta}
$$

for $1 \leq i \leq N$. Note that (7.2) holds iff $0=\sum_{i=1}^{N} d_{i} \tau_{i}$ iff $d_{i}=0$ for $1 \leq i \leq N$. For $1 \leq i \leq N$ we simplify (7.5) using

$$
\eta_{N-i+1}\left(a_{0}\right)=\eta_{N-i}\left(a_{0}\right)\left(a_{0}-b_{N-i}\right)
$$

and

$$
\left[\begin{array}{c}
N \\
i-1
\end{array}\right]_{\vartheta}=\left[\begin{array}{c}
N \\
i
\end{array}\right]_{\vartheta} \frac{\vartheta_{i}}{\vartheta_{N-i+1}}, \quad\left[\begin{array}{c}
N+1 \\
i
\end{array}\right]_{\vartheta}=\left[\begin{array}{c}
N \\
i
\end{array}\right]_{\vartheta} \frac{\vartheta_{N+1}}{\vartheta_{N-i+1}}
$$

We find that $d_{i}$ is equal to

$$
\frac{\eta_{N-i}\left(a_{0}\right)}{\vartheta_{N-i+1}}\left[\begin{array}{c}
N \\
i
\end{array}\right]_{\vartheta}
$$

times

$$
\left(a_{i}-b_{N}\right) \vartheta_{N-i+1}+\left(a_{0}-b_{N-i}\right)\left(\vartheta_{i}-\vartheta_{N+1}\right)
$$

for $1 \leq i \leq N$. Therefore, (7.2) holds if and only if

$$
\eta_{N-i}\left(a_{0}\right)=0 \quad \text { or } \quad\left(a_{i}-b_{N}\right) \vartheta_{N-i+1}=\left(a_{0}-b_{N-i}\right)\left(\vartheta_{N+1}-\vartheta_{i}\right)
$$

for $1 \leq i \leq N$. Replacing $i$ by $N-i$, we see that (7.2) holds if and only if

$$
\eta_{i}\left(a_{0}\right)=0 \quad \text { or } \quad\left(a_{N-i}-b_{N}\right) \vartheta_{i+1}=\left(a_{0}-b_{i}\right)\left(\vartheta_{N+1}-\vartheta_{N-i}\right)
$$

for $0 \leq i \leq N-1$. The result follows in view of Definition 4.1.

(i) $\Leftrightarrow$ (iii) Similar to the proof of (i) $\Leftrightarrow$ (ii). 
Our next general goal is to solve the equations in Proposition 7.3(ii), (iii). The main solution will involve a type of sequence, said to be recurrent.

\section{Recurrent sequences}

Throughout this section let $n$ denote an integer at least 2 , or $\infty$. let $\left\{a_{i}\right\}_{i=0}^{n}$ denote scalars in $\mathbb{F}$.

Definition 8.1 (see [30, Definition 8.2]). Let $\beta, \gamma, \varrho$ denote scalars in $\mathbb{F}$.

(i) The sequence $\left\{a_{i}\right\}_{i=0}^{n}$ is said to be $(\beta, \gamma, \varrho)$-recurrent whenever

$$
a_{i-1}^{2}-\beta a_{i-1} a_{i}+a_{i}^{2}-\gamma\left(a_{i-1}+a_{i}\right)=\varrho
$$

for $1 \leq i \leq n$.

(ii) The sequence $\left\{a_{i}\right\}_{i=0}^{n}$ is said to be $(\beta, \gamma)$-recurrent whenever

$$
a_{i-1}-\beta a_{i}+a_{i+1}=\gamma
$$

for $1 \leq i \leq n-1$.

(iii) The sequence $\left\{a_{i}\right\}_{i=0}^{n}$ is said to be $\beta$-recurrent whenever

$$
a_{i-2}-(\beta+1) a_{i-1}+(\beta+1) a_{i}-a_{i+1}
$$

is zero for $2 \leq i \leq n-1$.

(iv) The sequence $\left\{a_{i}\right\}_{i=0}^{n}$ is said to be recurrent whenever there exists $\beta \in \mathbb{F}$ such that $\left\{a_{i}\right\}_{i=0}^{n}$ is $\beta$-recurrent.

Lemma 8.2. The following are equivalent:

(i) the sequence $\left\{a_{i}\right\}_{i=0}^{n}$ is recurrent;

(ii) there exists $\beta \in \mathbb{F}$ such that $\left\{a_{i}\right\}_{i=0}^{n}$ is $\beta$-recurrent.

Proof. By Definition 8.1.

Lemma 8.3. For $\beta \in \mathbb{F}$ the following are equivalent:

(i) the sequence $\left\{a_{i}\right\}_{i=0}^{n}$ is $\beta$-recurrent;

(ii) there exists $\gamma \in \mathbb{F}$ such that $\left\{a_{i}\right\}_{i=0}^{n}$ is $(\beta, \gamma)$-recurrent.

Proof. (i) $\Rightarrow$ (ii) For $2 \leq i \leq n-1$, the expression (8.3) is zero by assumption, so

$$
a_{i-2}-\beta a_{i-1}+a_{i}=a_{i-1}-\beta a_{i}+a_{i+1} .
$$

The left-hand side of (8.2) is independent of $i$, and the result follows.

(ii) $\Rightarrow$ (i) For $2 \leq i \leq n-1$, subtract the equation (8.2) at $i$ from the corresponding equation obtained by replacing $i$ by $i-1$, to find (8.3) is zero.

Lemma 8.4. The following $(i)$, (ii) hold for all $\beta, \gamma \in \mathbb{F}$.

(i) Suppose $\left\{a_{i}\right\}_{i=0}^{n}$ is $(\beta, \gamma)$-recurrent. Then there exists $\varrho \in \mathbb{F}$ such that $\left\{a_{i}\right\}_{i=0}^{n}$ is $(\beta, \gamma, \varrho)$ recurrent.

(ii) Suppose $\left\{a_{i}\right\}_{i=0}^{n}$ is $(\beta, \gamma, \varrho)$-recurrent, and that $a_{i-1} \neq a_{i+1}$ for $1 \leq i \leq n-1$. Then $\left\{a_{i}\right\}_{i=0}^{n}$ is $(\beta, \gamma)$-recurrent. 
Proof. Let $p_{i}$ denote the expression on the left in (8.1), and observe

$$
p_{i}-p_{i+1}=\left(a_{i-1}-a_{i+1}\right)\left(a_{i-1}-\beta a_{i}+a_{i+1}-\gamma\right)
$$

for $1 \leq i \leq n-1$. Assertions (i), (ii) are both routine consequences of this.

Definition 8.5. Assume that $\left\{a_{i}\right\}_{i=0}^{n}$ is recurrent. By a parameter triple for $\left\{a_{i}\right\}_{i=0}^{n}$ we mean a 3 -tuple $\beta, \gamma, \varrho$ of scalars in $\mathbb{F}$ such that $\left\{a_{i}\right\}_{i=0}^{n}$ is $(\beta, \gamma)$-recurrent and $(\beta, \gamma, \varrho)$-recurrent.

Note that a recurrent sequence has at least one parameter triple.

\section{Recurrent sequences in closed form}

In this section, we describe the recurrent sequences in closed form. Let $n$ denote an integer at least 2 , or $\infty$.

Lemma 9.1 (see [30, Lemma 9.2]). The recurrent sequences $\left\{a_{i}\right\}_{i=0}^{n}$ are described in the table below:

\begin{tabular}{c|c|c} 
case & $a_{i}$ & comments \\
\hline I & $\alpha_{1}+\alpha_{2} q^{i}+\alpha_{3} q^{-i}$ & $q \notin\{0,1,-1\}$ \\
II & $\alpha_{1}+\alpha_{2} i+\alpha_{3}\left(\begin{array}{c}i \\
2\end{array}\right)$ & \\
III & $\alpha_{1}+\alpha_{2}(-1)^{i}+\alpha_{3} i(-1)^{i}$ & $\operatorname{char}(\mathbb{F}) \neq 2$
\end{tabular}

In the above table $q, \alpha_{1}, \alpha_{2}, \alpha_{3}$ are scalars in $\mathbb{F}$.

Lemma 9.2. The following scalars $\beta, \gamma$, @ give a parameter triple for the recurrent sequence $\left\{a_{i}\right\}_{i=0}^{n}$ in Lemma 9.1 .

Case I:

$$
\beta=q+q^{-1}, \quad \gamma=-\alpha_{1}(q-1)^{2} q^{-1}, \quad \varrho=\alpha_{1}^{2}(q-1)^{2} q^{-1}-\alpha_{2} \alpha_{3}\left(q-q^{-1}\right)^{2} .
$$

Case II:

$$
\beta=2, \quad \gamma=\alpha_{3}, \quad \varrho=\alpha_{2}^{2}-\alpha_{2} \alpha_{3}-2 \alpha_{1} \alpha_{3} .
$$

Case III:

$$
\beta=-2, \quad \gamma=4 \alpha_{1}, \quad \varrho=\alpha_{3}^{2}-4 \alpha_{1}^{2} .
$$

Proof. This is routinely checked using Definition 8.5.

Lemma 9.3. Referring to Lemma 9.1, for $0 \leq i \leq n+1$ the sum $a_{0}+a_{1}+\cdots+a_{i-1}$ is given in the table below:

\begin{tabular}{c|c} 
case & $a_{0}+a_{1}+\cdots+a_{i-1}$ \\
\hline$I$ & $\alpha_{1} i+\alpha_{2} \frac{1-q^{i}}{1-q}+\alpha_{3} \frac{1-q^{-i}}{1-q^{-1}}$ \\
$I I$ & $\alpha_{1} i+\alpha_{2}\left(\begin{array}{l}i \\
2\end{array}\right)+\alpha_{3}\left(\begin{array}{l}i \\
3\end{array}\right)$ \\
III, $i$ even & $\alpha_{1} i-\alpha_{3} i / 2$ \\
III, $i$ odd & $\alpha_{1} i+\alpha_{2}+\alpha_{3}(i-1) / 2$
\end{tabular}

Proof. Use induction on $i$.

Note 9.4. Referring to Case III of the above table, the subcases $i$ even and $i$ odd can be handled in the following uniform way. For $0 \leq i \leq n+1$,

$$
a_{0}+a_{1}+\cdots+a_{i-1}=\frac{2 \alpha_{2}-\alpha_{3}+4 \alpha_{1} i+\left(\alpha_{3}-2 \alpha_{2}\right)(-1)^{i}-2 \alpha_{3} i(-1)^{i}}{4} .
$$




\section{Twin recurrent sequences}

Let $n$ denote an integer at least 2 , or $\infty$. Let $\left\{a_{i}\right\}_{i=0}^{n},\left\{b_{i}\right\}_{i=0}^{n}$ denote scalars in $\mathbb{F}$.

Definition 10.1. Assume that the sequences $\left\{a_{i}\right\}_{i=0}^{n},\left\{b_{i}\right\}_{i=0}^{n}$ are recurrent. These sequences are called twins whenever they have a parameter triple in common.

Lemma 10.2. Assume that the sequences $\left\{a_{i}\right\}_{i=0}^{n},\left\{b_{i}\right\}_{i=0}^{n}$ are recurrent. These sequences are twins if and only if they are related in the following way:

Case I:

$$
a_{i}=\alpha_{1}+\alpha_{2} q^{i}+\alpha_{3} q^{-i}, \quad b_{i}=\alpha_{1}+\alpha_{2}^{\prime} q^{i}+\alpha_{3}^{\prime} q^{-i}, \quad \alpha_{2}^{\prime} \alpha_{3}^{\prime}=\alpha_{2} \alpha_{3} .
$$

Case II:

$$
\begin{aligned}
& a_{i}=\alpha_{1}+\alpha_{2} i+\alpha_{3}\left(\begin{array}{l}
i \\
2
\end{array}\right), \quad b_{i}=\alpha_{1}^{\prime}+\alpha_{2}^{\prime} i+\alpha_{3}\left(\begin{array}{l}
i \\
2
\end{array}\right), \\
& \left(\alpha_{2}-\alpha_{2}^{\prime}\right)\left(\alpha_{2}+\alpha_{2}^{\prime}-\alpha_{3}\right)=2\left(\alpha_{1}-\alpha_{1}^{\prime}\right) \alpha_{3} .
\end{aligned}
$$

Case III:

$$
\begin{aligned}
& a_{i}=\alpha_{1}+\alpha_{2}(-1)^{i}+\alpha_{3} i(-1)^{i}, \quad b_{i}=\alpha_{1}+\alpha_{2}^{\prime}(-1)^{i}+\alpha_{3}^{\prime} i(-1)^{i}, \\
& \alpha_{3}^{\prime}=\alpha_{3} \quad \text { or } \quad \alpha_{3}^{\prime}=-\alpha_{3} .
\end{aligned}
$$

Proof. First assume that the sequences $\left\{a_{i}\right\}_{i=0}^{n},\left\{b_{i}\right\}_{i=0}^{n}$ are related in the specified way. Then these sequences share the parameter triple $\beta, \gamma, \varrho$ from Lemma 9.2. Therefore these sequences are twins. Next assume that the sequences $\left\{a_{i}\right\}_{i=0}^{n},\left\{b_{i}\right\}_{i=0}^{n}$ are twins. It follows from Definition 8.1 and Lemma 9.1 that they are related in the specified way.

\section{A characterization of twin recurrent sequences}

In this section we explain what twin recurrent sequences have to do with the equations in Proposition 7.3. Let $n$ denote an integer at least 2 , or $\infty$. Let $\left\{a_{i}\right\}_{i=0}^{n},\left\{b_{i}\right\}_{i=0}^{n}$ denote scalars in $\mathbb{F}$.

Definition 11.1. For $0 \leq i \leq j \leq n$ let $E(i, j)$ denote the equation

$$
\left(a_{0}+\cdots+a_{i}-b_{0}-\cdots-b_{i}\right)\left(a_{j-i}-b_{j}\right)=\left(a_{0}-b_{i}\right)\left(a_{j-i}+\cdots+a_{j}-b_{j-i}-\cdots-b_{j}\right) .
$$

Lemma 11.2. The equations $E(0, j)$ and $E(j, j)$ hold for $0 \leq j \leq n$.

Proof. This is routinely checked.

Proposition 11.3. Assume that the sequences $\left\{a_{i}\right\}_{i=0}^{n},\left\{b_{i}\right\}_{i=0}^{n}$ are recurrent and twins. Then $E(i, j)$ holds for $0 \leq i \leq j \leq n$.

Proof. This is routinely verified for each Case I-III in Lemma 10.2. To carry out the verification, use the formulas in Lemma 9.3.

In the next two lemmas, we give some additional solutions for the equations $E(i, j)$ in Definition 11.1.

Lemma 11.4. Assume that $a_{i}=b_{i-1}$ for $1 \leq i \leq n$. Then $E(i, j)$ holds for $0 \leq i \leq j \leq n$.

Proof. For $0 \leq i \leq j \leq n$, each side of $E(i, j)$ is equal to $\left(a_{0}-b_{i}\right)\left(a_{j-i}-b_{j}\right)$. 
Lemma 11.5. Pick $\theta \in \mathbb{F}$ and assume

$$
\begin{aligned}
& a_{i}=\theta, \quad b_{i}=\theta, \quad 1 \leq i \leq n-1, \\
& \left(\theta-a_{0}\right)\left(\theta-a_{n}\right)=\left(\theta-b_{0}\right)\left(\theta-b_{n}\right) \quad \text { if } n \neq \infty .
\end{aligned}
$$

Then $E(i, j)$ holds for $0 \leq i \leq j \leq n$.

Proof. By Lemma 11.2 it suffices to verify $E(i, j)$ for $1 \leq i<j \leq n$. Let $i, j$ be given. For $j<n$, each side of $E(i, j)$ is zero. For $n \neq \infty$ and $j=n$, the equation $E(i, j)$ becomes

$$
\left(a_{0}-b_{0}\right)\left(\theta-b_{n}\right)=\left(a_{0}-\theta\right)\left(a_{n}-b_{n}\right)
$$

which is a reformulation of (11.1).

Proposition 11.6. Assume that $a_{1} \neq b_{0}$ and $a_{1} \neq b_{1}$. Further assume that $E(i, j)$ holds for $1 \leq i \leq 2$ and $i+1 \leq j \leq n$. Then the sequences $\left\{a_{i}\right\}_{i=0}^{n},\left\{b_{i}\right\}_{i=0}^{n}$ are recurrent and twins.

Proof. Using $E(1,2)$,

$$
\left(a_{0}-b_{1}\right)\left(a_{2}-b_{1}\right)=\left(b_{0}-a_{1}\right)\left(b_{2}-a_{1}\right) .
$$

Since $a_{1} \neq b_{1}$, there exists a unique pair $\beta, \gamma$ of scalars in $\mathbb{F}$ such that

$$
a_{0}-\beta a_{1}+a_{2}=\gamma, \quad b_{0}-\beta b_{1}+b_{2}=\gamma .
$$

Using these equations we eliminate $a_{2}, b_{2}$ in (11.2):

$$
\left(a_{0}-b_{1}\right)\left(\gamma+\beta a_{1}-a_{0}-b_{1}\right)=\left(b_{0}-a_{1}\right)\left(\gamma+\beta b_{1}-b_{0}-a_{1}\right) .
$$

In this equation we rearrange terms to get

$$
a_{0}^{2}-\beta a_{0} a_{1}+a_{1}^{2}-\gamma\left(a_{0}+a_{1}\right)=b_{0}^{2}-\beta b_{0} b_{1}+b_{1}^{2}-\gamma\left(b_{0}+b_{1}\right) .
$$

Let $\varrho$ denote this common value. We show that each of $\left\{a_{i}\right\}_{i=0}^{n},\left\{b_{i}\right\}_{i=0}^{n}$ is $(\beta, \gamma)$-recurrent and $(\beta, \gamma, \varrho)$-recurrent. To do this, we show that for $2 \leq j \leq n$, each of $\left\{a_{i}\right\}_{i=0}^{j},\left\{b_{i}\right\}_{i=0}^{j}$ is $(\beta, \gamma)$-recurrent and $(\beta, \gamma, \varrho)$-recurrent. We will use induction on $j$. First assume that $j=2$. By construction $\left\{a_{i}\right\}_{i=0}^{2}$ is $(\beta, \gamma)$-recurrent. By construction and Lemma 8.4(i), the sequence $\left\{a_{i}\right\}_{i=0}^{2}$ is $(\beta, \gamma, \varrho)$-recurrent. Similarly $\left\{b_{i}\right\}_{i=0}^{2}$ is $(\beta, \gamma)$-recurrent and $(\beta, \gamma, \varrho)$-recurrent. We are done for $j=2$. Next assume that $j \geq 3$. By $E(1, j)$,

$$
\left(a_{0}+a_{1}-b_{0}-b_{1}\right)\left(a_{j-1}-b_{j}\right)=\left(a_{0}-b_{1}\right)\left(a_{j-1}+a_{j}-b_{j-1}-b_{j}\right) .
$$

By $E(2, j)$,

$$
\begin{aligned}
& \left(a_{0}+a_{1}+a_{2}-b_{0}-b_{1}-b_{2}\right)\left(a_{j-2}-b_{j}\right) \\
& \quad=\left(a_{0}-b_{2}\right)\left(a_{j-2}+a_{j-1}+a_{j}-b_{j-2}-b_{j-1}-b_{j}\right) .
\end{aligned}
$$

The equations (11.3), (11.4) give a linear system in the unknowns $a_{j}, b_{j}$. For this system the coefficient matrix is

$$
C=\left(\begin{array}{cc}
a_{0}-b_{1} & a_{1}-b_{0} \\
a_{0}-b_{2} & a_{1}+a_{2}-b_{0}-b_{1}
\end{array}\right) .
$$

We have

$$
\operatorname{det}(C)=\left(a_{0}-b_{1}\right)\left(a_{1}+a_{2}-b_{0}-b_{1}\right)-\left(a_{0}-b_{2}\right)\left(a_{1}-b_{0}\right) .
$$


In the above equation we simplify the right-hand side using (11.2), to obtain

$$
\operatorname{det}(C)=\left(a_{1}-b_{0}\right)\left(a_{1}-b_{1}\right) .
$$

Therefore $\operatorname{det}(C) \neq 0$, so the system (11.3), (11.4) has a unique solution for $a_{j}, b_{j}$. We now describe the solution. By induction the sequences $\left\{a_{i}\right\}_{i=0}^{j-1},\left\{b_{i}\right\}_{i=0}^{j-1}$ are $(\beta, \gamma)$-recurrent and $(\beta, \gamma, \varrho)$-recurrent. Define $a_{j}^{\prime}, b_{j}^{\prime}$ such that

$$
a_{j-2}-\beta a_{j-1}+a_{j}^{\prime}=\gamma, \quad b_{j-2}-\beta b_{j-1}+b_{j}^{\prime}=\gamma .
$$

Consider the two sequences

$$
\begin{aligned}
& a_{0}, a_{1}, \ldots, a_{j-1}, a_{j}^{\prime} \\
& b_{0}, b_{1}, \ldots, b_{j-1}, b_{j}^{\prime} .
\end{aligned}
$$

By construction, each of (11.5), (11.6) is $(\beta, \gamma)$-recurrent. By construction and Lemma 8.4(i), each of $(11.5),(11.6)$ is $(\beta, \gamma, \varrho)$-recurrent. We show that $a_{j}=a_{j}^{\prime}$ and $b_{j}=b_{j}^{\prime}$. The sequences (11.5), (11.6) are recurrent and twins, so by Proposition 11.3 they satisfy $E(1, j)$ and $E(2, j)$. Therefore, the equations (11.3), (11.4) still hold if we replace $a_{j}, b_{j}$ by $a_{j}^{\prime}, b_{j}^{\prime}$. We mentioned earlier that the system (11.3), (11.4) has a unique solution for $a_{j}, b_{j}$. By these comments $a_{j}=a_{j}^{\prime}$ and $b_{j}=b_{j}^{\prime}$. Consequently each of $\left\{a_{i}\right\}_{i=0}^{j},\left\{b_{i}\right\}_{i=0}^{j}$ is $(\beta, \gamma)$-recurrent and $(\beta, \gamma, \varrho)$-recurrent. The above argument shows that each of $\left\{a_{i}\right\}_{i=0}^{n},\left\{b_{i}\right\}_{i=0}^{n}$ is $(\beta, \gamma)$-recurrent and $(\beta, \gamma, \varrho)$-recurrent.

Lemma 11.7. Assume that $a_{i-1}=b_{i}$ for $1 \leq i \leq n$. Then the equation $E(1, j)$ holds for $1 \leq j \leq n$. However, in general it is not the case that $E(i, j)$ holds for $0 \leq i \leq j \leq n$.

Proof. For $0 \leq i \leq j \leq n$ the equation $E(i, j)$ becomes

$$
\left(a_{i}-b_{0}\right)\left(a_{j-i}-b_{j}\right)=\left(a_{0}-b_{i}\right)\left(a_{j}-b_{j-i}\right) .
$$

If $i=1$ then each side of (11.7) is zero, so $E(1, j)$ holds. Assume that $n=3$ and

$$
a_{0}=0=b_{1}, \quad a_{1}=1=b_{2}, \quad a_{2}=0=b_{3}, \quad a_{3}=1, \quad b_{0}=0 .
$$

Then (11.7) fails for $i=2$ and $j=3$.

\section{The classification of the double lowering data}

Recall the data $\left\{a_{i}\right\}_{i=0}^{N-1},\left\{b_{i}\right\}_{i=0}^{N-1}$ from (2.1). In this section we obtain necessary and sufficient conditions for this data to be double lowering. In view of Lemma 6.2 we assume $N \geq 3$.

Theorem 12.1. Let $N$ denote an integer at least 3 , or $\infty$. Let

$$
\left\{a_{i}\right\}_{i=0}^{N-1}, \quad\left\{b_{i}\right\}_{i=0}^{N-1}
$$

denote scalars in $\mathbb{F}$ such that

$$
a_{0}+a_{1}+\cdots+a_{i-1} \neq b_{0}+b_{1}+\cdots+b_{i-1}, \quad 1 \leq i \leq N .
$$

Then the data (12.1) is double lowering if and only if at least one of the following $(i)-(i v)$ holds:

(i) $a_{i-1}=b_{i}$ for $1 \leq i \leq N-1$;

(ii) $a_{i}=b_{i-1}$ for $1 \leq i \leq N-1$; 
(iii) there exists $\theta \in \mathbb{F}$ such that

$$
\begin{aligned}
& a_{0} \neq \theta, \quad b_{0} \neq \theta, \quad a_{i}=\theta, \quad b_{i}=\theta, \quad 1 \leq i \leq N-2, \\
& \frac{\theta-a_{N-1}}{\theta-b_{0}}=\frac{\theta-b_{N-1}}{\theta-a_{0}} \quad \text { if } N \neq \infty .
\end{aligned}
$$

(iv) the sequences (12.1) are recurrent and twins.

Proof. First assume that at least one of (i)-(iii) holds. Then (12.1) is double lowering, by Lemmas 6.4, 6.5, 6.6. Next assume that (iv) holds and $N \neq \infty$. By Proposition 11.3 (with $n=N-1)$ the equations $E(i, j)$ hold for $0 \leq i \leq j \leq N-1$. We delete $a_{N-1}, b_{N-1}$ from (12.1) and consider the data

$$
\left\{a_{i}\right\}_{i=0}^{N-2}, \quad\left\{b_{i}\right\}_{i=0}^{N-2} .
$$

By Lemma 6.2 and induction on $N$, we may assume that the sequences (12.3) are double lowering. By Proposition 7.3(i), (ii) (with $N$ replaced by $N-1$ ) we find that (12.1) is double lowering. Next assume that (iv) holds and $N=\infty$. Then for all integers $j \geq 2$ the sequences $\left\{a_{i}\right\}_{i=0}^{j}$, $\left\{b_{i}\right\}_{i=0}^{j}$ are recurrent and twins. Consequently the data $\left\{a_{i}\right\}_{i=0}^{j},\left\{b_{i}\right\}_{i=0}^{j}$ is double lowering. Therefore the data $\left\{a_{i}\right\}_{i=0}^{\infty},\left\{b_{i}\right\}_{i=0}^{\infty}$ is double lowering. We are done in one direction.

We now reverse the direction. Next assume that (12.1) is double lowering. We break the argument into cases.

Case $a_{0}=b_{1}$. We show that (i) holds. We have $\eta_{i}\left(a_{0}\right) \neq 0$ for $0 \leq i \leq 1$. By assumption the data (12.1) is double lowering, so the data $\left\{a_{i}\right\}_{i=0}^{j},\left\{b_{i}\right\}_{i=0}^{j}$ is double lowering for $1 \leq j \leq N-1$. Applying Proposition 7.3(i), (ii) repeatedly (with $N$ replaced by $2,3, \ldots, N-1$ ) we find that $E(1, j)$ holds for $2 \leq j \leq N-1$. Using these equations and (12.2) we routinely obtain $a_{j-1}=b_{j}$ for $2 \leq j \leq N-1$. This and $a_{0}=b_{1}$ implies (i).

Case $a_{1}=b_{0}$. Interchanging the roles of $\left\{a_{i}\right\}_{i=0}^{N-1},\left\{b_{i}\right\}_{i=0}^{N-1}$ in the previous case, we find that (ii) holds.

Case $a_{0} \neq b_{1}, a_{1} \neq b_{0}, a_{1}=b_{1}$. We show that (iii) holds. Define $\theta=a_{1}=b_{1}$, and note that $a_{0} \neq \theta, b_{0} \neq \theta$. We have $\eta_{i}\left(a_{0}\right) \neq 0$ for $0 \leq i \leq 2$. By assumption the data (12.1) is double lowering, so the data $\left\{a_{i}\right\}_{i=0}^{j},\left\{b_{i}\right\}_{i=0}^{j}$ is double lowering for $1 \leq j \leq N-1$. Applying Proposition $7.3(\mathrm{i})$, (ii) repeatedly (with $N$ replaced by $2,3, \ldots, N-1$ ) we find that $E(i, j)$ holds for $1 \leq i \leq 2$ and $i+1 \leq j \leq N-1$. Next we show that $a_{k}=b_{k}=\theta$ for $2 \leq k \leq N-2$. We will use induction on $k$. Assume $N \geq 4$; otherwise there is nothing to prove. Using $E(1,2), E(1,3)$ and $a_{0} \neq b_{1}$ we obtain

$$
\begin{aligned}
& a_{2}=b_{2} \zeta+\theta(1-\zeta), \\
& a_{3}=b_{3} \zeta+b_{2}\left(1-\zeta^{2}\right)-\theta \zeta(1-\zeta),
\end{aligned}
$$

where

$$
\zeta=\frac{b_{0}-\theta}{a_{0}-\theta} .
$$

In the equation $E(2,3)$ we eliminate $a_{2}, a_{3}$ using (12.4), (12.5). We evaluate the result using (12.2) (with $i=3$ ), to obtain $b_{2}=\theta$. In (12.4) we set $b_{2}=\theta$ to obtain $a_{2}=\theta$. Next assume that $3 \leq k \leq N-2$. By induction each of $a_{2}, a_{3}, \ldots, a_{k-1}, b_{2}, b_{3}, \ldots, b_{k-1}$ is equal to $\theta$. Using this we evaluate $E(1, k), E(1, k+1)$ to obtain

$$
\begin{aligned}
& a_{k}=b_{k} \zeta+\theta(1-\zeta), \\
& a_{k+1}=b_{k+1} \zeta+b_{k}\left(1-\zeta^{2}\right)-\theta \zeta(1-\zeta) .
\end{aligned}
$$


Using (12.7), (12.8) we evaluate $E(2, k+1)$ to obtain $b_{k}=\theta$. In (12.7) we set $b_{k}=\theta$ to obtain $a_{k}=\theta$. We have shown that $a_{k}=b_{k}=\theta$ for $2 \leq k \leq N-2$. Now for $N \neq \infty$ we use $E(1, N-1)$ to obtain

$$
a_{N-1}=b_{N-1} \zeta+\theta(1-\zeta)
$$

Evaluating this using (12.6) we obtain

$$
\frac{\theta-a_{N-1}}{\theta-b_{0}}=\frac{\theta-b_{N-1}}{\theta-a_{0}}
$$

We have shown that (iii) holds.

Case $a_{0} \neq b_{1}, a_{1} \neq b_{0}, a_{1} \neq b_{1}$. We show that (iv) holds. Using Proposition 7.3(i), (ii) as in the previous case, we find that $E(i, j)$ holds for $1 \leq i \leq 2$ and $i+1 \leq j \leq N-1$. Now by Proposition 11.6 (with $n=N-1$ ), the sequences (12.1) are recurrent and twins. We have shown that (iv) holds.

\section{$13 \mathcal{L}$ and $\Delta$ for twin recurrent data}

Our goal for the rest of the paper is to give a comprehensive description of $\mathcal{L}$ and $\Delta$ for twin recurrent data. We will focus on Case I in Lemma 10.2, or more precisely, an adjusted version of this case as described in Section 3.

For the rest of this paper we assume that $N$ is an integer at least 2 , or $\infty$. Recall the double lowering space $\mathcal{L}$ for the data (2.1). For the rest of this paper, fix nonzero $a, b, q \in \mathbb{F}$ and assume

$$
a_{i}=a q^{i}+a^{-1} q^{-i}, \quad b_{i}=b q^{i}+b^{-1} q^{-i}
$$

for $0 \leq i \leq N-1$. By $(2.2)$ we have $a \neq b$ and

$$
q^{i} \neq 1, \quad a b q^{i-1} \neq 1, \quad 1 \leq i \leq N
$$

Note 13.1. The data $\left\{a_{i}\right\}_{i=0}^{N-1},\left\{b_{i}\right\}_{i=0}^{N-1}$ is unchanged if we replace

$$
q \mapsto q^{-1}, \quad a \mapsto a^{-1}, \quad b \mapsto b^{-1} .
$$

Lemma 13.2. The sequences (2.1) are recurrent and twins.

Proof. By Lemmas 9.1 and 10.2.

Corollary 13.3. The data (2.1) is double lowering.

Proof. By Lemma 13.2 along with Lemma 6.2 and Theorem 12.1(iv).

Our next general goal is to describe the polynomials $\left\{\tau_{i}\right\}_{i=0}^{N-1},\left\{\eta_{i}\right\}_{i=0}^{N-1}$ from (2.3), (2.4), the parameters $\left\{\vartheta_{i}\right\}_{i=0}^{N}$ from Definition 4.1, and the map $\Delta$ from (2.5).

We mention some formulas for later use.

Lemma 13.4. For $1 \leq i \leq N-1$,

$$
\begin{array}{ll}
q a_{i}-a_{i-1}=\left(q-q^{-1}\right) a q^{i}, & q b_{i}-b_{i-1}=\left(q-q^{-1}\right) b q^{i}, \\
a_{i}-a_{i-1}=(q-1)\left(a q^{i-1}-a^{-1} q^{-i}\right), & b_{i}-b_{i-1}=(q-1)\left(b q^{i-1}-b^{-1} q^{-i}\right), \\
a_{i}-q a_{i-1}=\left(1-q^{2}\right) a^{-1} q^{-i}, & b_{i}-q b_{i-1}=\left(1-q^{2}\right) b^{-1} q^{-i} .
\end{array}
$$

Proof. Use (13.1). 
Lemma 13.5. For $0 \leq i \leq N$,

$$
\begin{aligned}
& a_{0}+a_{1}+\cdots+a_{i-1}=\frac{1-q^{i}}{1-q}\left(a+a^{-1} q^{1-i}\right), \\
& b_{0}+b_{1}+\cdots+b_{i-1}=\frac{1-q^{i}}{1-q}\left(b+b^{-1} q^{1-i}\right) .
\end{aligned}
$$

Proof. Use (13.1).

Next we describe $\left\{\vartheta_{i}\right\}_{i=0}^{N}$. We give two versions.

Lemma 13.6. For $0 \leq i \leq N$,

(i) $\vartheta_{i}=\frac{1-q^{i}}{1-q} \frac{1-a b q^{i-1}}{1-a b} q^{1-i}$;

(ii) $\vartheta_{i}=\frac{1-q^{-i}}{1-q^{-1}} \frac{1-a^{-1} b^{-1} q^{1-i}}{1-a^{-1} b^{-1}} q^{i-1}$.

Proof. (i) By Definition 4.1 and Lemma 13.5. (ii) By Note 13.1 and (i) above.

We mention some formulas for later use.

Lemma 13.7. For $0 \leq i \leq N-1$,

$$
\begin{aligned}
& q \vartheta_{i+1}-\vartheta_{i}=\frac{q+a b-(q+1) a b q^{i}}{1-a b}, \\
& \vartheta_{i+1}-\vartheta_{i}=\frac{q^{-i}-a b q^{i}}{1-a b}, \\
& \vartheta_{i+1}-q \vartheta_{i}=\frac{(1+q) q^{-i}-q-a b}{1-a b} .
\end{aligned}
$$

Proof. Use Lemma 13.6.

We recall some notation. For an element $\alpha$ in any algebra, define

$$
(\alpha ; q)_{i}=(1-\alpha)(1-\alpha q) \cdots\left(1-\alpha q^{i-1}\right), \quad i \in \mathbb{N} .
$$

We interpret $(\alpha ; q)_{0}=1$. We remark that for $j \geq i \geq 0$,

$$
\left(q^{-j} ; q\right)_{i}(q ; q)_{j-i}=(-1)^{i}(q ; q)_{j} q^{\left(\begin{array}{c}
i \\
2
\end{array}\right)} q^{-i j} .
$$

Lemma 13.8. For $0 \leq i \leq N$,

$$
\vartheta_{1} \vartheta_{2} \cdots \vartheta_{i}=\frac{(q ; q)_{i}(a b ; q)_{i} q^{-\left(\begin{array}{c}
i \\
2
\end{array}\right)}}{(1-q)^{i}(1-a b)^{i}}
$$

For $0 \leq i \leq j \leq N$,

$$
\vartheta_{j} \vartheta_{j-1} \cdots \vartheta_{j-i+1}=\frac{\left(q^{-j} ; q\right)_{i}\left(a^{-1} b^{-1} q^{1-j} ; q\right)_{i} q^{i(j-i)} q^{\left(\begin{array}{c}
i \\
2
\end{array}\right)}}{\left(1-q^{-1}\right)^{i}\left(1-a^{-1} b^{-1}\right)^{i}} .
$$

Proof. To obtain (13.3), use Lemma 13.6(i). To obtain (13.4), use Lemma 13.6(ii).

Lemma 13.9. For $0 \leq i \leq j \leq N$,

$$
\left[\begin{array}{c}
j \\
i
\end{array}\right]_{\vartheta}=\frac{\left(q^{-j} ; q\right)_{i}\left(a^{-1} b^{-1} q^{1-j} ; q\right)_{i} q^{i j} a^{i} b^{i}}{(q ; q)_{i}(a b ; q)_{i}} .
$$


Proof. Evaluate (5.3) using Lemma 13.8.

We comment on the notation. Let $y$ denote an indeterminate. Let $\mathbb{F}\left[y, y^{-1}\right]$ denote the algebra consisting of the Laurent polynomials in $y$ that have all coefficients in $\mathbb{F}$. This algebra has an automorphism that sends $y \mapsto y^{-1}$. An element of $\mathbb{F}\left[y, y^{-1}\right]$ that is fixed by the automorphism is called symmetric. The symmetric elements form a subalgebra of $\mathbb{F}\left[y, y^{-1}\right]$ called its symmetric part. There exists an injective algebra homomorphism $\iota: \mathbb{F}[x] \rightarrow \mathbb{F}\left[y, y^{-1}\right]$ that sends $x \mapsto$ $y+y^{-1}$. The image of $\mathbb{F}[x]$ under $\iota$ is the symmetric part of $\mathbb{F}\left[y, y^{-1}\right]$. Via $\iota$ we identify $\mathbb{F}[x]$ with the symmetric part of $\mathbb{F}\left[y, y^{-1}\right]$.

Lemma 13.10. For $0 \leq i \leq N$,

(i) $\tau_{i}=(-1)^{i} a^{-i} q^{-\left(\begin{array}{c}i \\ 2\end{array}\right)(a y ; q)_{i}\left(a y^{-1} ; q\right)_{i}}$;

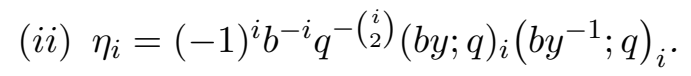

In the above lines $x=y+y^{-1}$.

Proof. (i) We use (2.3) and (13.1). For $0 \leq j \leq i-1$,

$$
x-a_{j}=y+y^{-1}-a q^{j}-a^{-1} q^{-j}=-a^{-1} q^{-j}\left(1-a y q^{j}\right)\left(1-a y^{-1} q^{j}\right) .
$$

The result follows. (ii) Similar to the proof of (i) above.

Lemma 13.11. For $0 \leq i \leq N$
(i) $\tau_{i}\left(b_{0}\right)=(-1)^{i} a^{-i} q^{-\left(\begin{array}{c}i \\ 2\end{array}\right)(a b ; q)_{i}\left(a b^{-1} ; q\right)_{i}}$;
(ii) $\eta_{i}\left(a_{0}\right)=(-1)^{i} b^{-i} q^{-\left(\begin{array}{c}i \\ 2\end{array}\right)}(a b ; q)_{i}\left(a^{-1} b ; q\right)_{i}$.

Proof. (i) Set $y=b$ in Lemma 13.10(i), and use $b_{0}=b+b^{-1}$. (ii) Similar to the proof of (i) above.

Our data is double lowering, so $\mathcal{L} \neq 0$. For the rest of the paper, let $\psi \in \mathcal{L}$ be normalized. The maps $\Delta, \psi$ are related by (5.5), (5.6). Our next goal is to interpret (5.5), (5.6) using the $q$-exponential function. This function is defined as follows. For locally nilpotent $T \in \operatorname{End}(V)$,

$$
\exp _{q}(T)=\sum_{i=0}^{N} \frac{q^{\left(\begin{array}{c}
i \\
2
\end{array}\right)}(1-q)^{i} T^{i}}{(q ; q)_{i}} .
$$

The map $\exp _{q}(T)$ is invertible; its inverse is

$$
\exp _{q^{-1}}(-T)=\sum_{i=0}^{N} \frac{(-1)^{i}(1-q)^{i} T^{i}}{(q ; q)_{i}}
$$

Lemma 13.12. For locally nilpotent $T \in \operatorname{End}(V)$,

$$
(1-(q-1) T) \exp _{q}(q T)=\exp _{q}(T) .
$$

Proof. To verify this equation, for $0 \leq i \leq N$ compare the coefficient of $T^{i}$ on each side.

Proposition 13.13. We have

$$
\Delta=\exp _{q}\left(a^{-1} \xi \psi\right) \exp _{q^{-1}}\left(-b^{-1} \xi \psi\right)
$$

where $\xi=1-a b$. 
Proof. For $0 \leq j \leq N$ we compare the coefficient of $\psi^{j}$ on each side of (13.7). For the left-hand side these coefficients are obtained from (5.5). We require

$$
\frac{\eta_{j}\left(a_{0}\right)}{\vartheta_{1} \vartheta_{2} \cdots \vartheta_{j}}=\sum_{i=0}^{j} \frac{q^{\left(\begin{array}{l}
i \\
2
\end{array}\right)} a^{-i}(1-q)^{i} \xi^{i}}{(q ; q)_{i}} \frac{(-1)^{j-i} b^{i-j}(1-q)^{j-i} \xi^{j-i}}{(q ; q)_{j-i}} .
$$

By (13.3) and the construction,

$$
\vartheta_{1} \vartheta_{2} \cdots \vartheta_{j}=(q ; q)_{j}(a b ; q)_{j} q^{-\left(\begin{array}{l}
j \\
2
\end{array}\right)(1-q)^{-j} \xi^{-j} .}
$$

By Lemma 13.11(ii),

$$
\eta_{j}\left(a_{0}\right)=(-1)^{j} b^{-j} q^{-\left(\begin{array}{c}
j \\
2
\end{array}\right)(a b ; q)_{j}\left(a^{-1} b ; q\right)_{j} .}
$$

Using these comments and (13.2), the equation (13.8) becomes

$$
\left(z q^{-j} ; q\right)_{j}=\sum_{i=0}^{j} \frac{\left(q^{-j} ; q\right)_{i} z^{i}}{(q ; q)_{i}}
$$

where $z=a^{-1} b q^{j}$. Basic hypergeometric series are discussed in $[13,25]$. In (13.9) the sum on the right is the basic hypergeometric series

$$
{ }_{1} \phi_{0}\left(\begin{array}{c|c}
q^{-j} & q ; z) \\
- &
\end{array}\right.
$$

This observation reveals that (13.9) is an instance of the $q$-binomial theorem [13, Section 1.3]. The result follows.

Proposition 13.13 gives a factorization of $\Delta$. We now investigate the factors.

Lemma 13.14. For $0 \leq i \leq N$,

$$
\begin{aligned}
& \exp _{q^{-1}}\left(-a^{-1} \xi \psi\right) \eta_{i}=\exp _{q^{-1}}\left(-b^{-1} \xi \psi\right) \tau_{i} \\
& \exp _{q}\left(b^{-1} \xi \psi\right) \eta_{i}=\exp _{q}\left(a^{-1} \xi \psi\right) \tau_{i}
\end{aligned}
$$

where $\xi=1-a b$.

Proof. By (13.7) and the comments above Lemma 13.12,

$$
\exp _{q^{-1}}\left(-a^{-1} \xi \psi\right) \Delta=\exp _{q^{-1}}\left(-b^{-1} \xi \psi\right)
$$

To obtain (13.10), apply each side of (13.12) to $\tau_{i}$ and evaluate the result using (2.5). For the equation (13.7), the two factors on the right commute; swapping these factors and proceeding as above,

$$
\exp _{q}\left(b^{-1} \xi \psi\right) \Delta=\exp _{q}\left(a^{-1} \xi \psi\right)
$$

To obtain (13.11), apply each side of (13.13) to $\tau_{i}$ and evaluate the result using (2.5).

Definition 13.15. For $0 \leq i \leq N$ let $w_{i}$ (resp. $w_{i}^{\prime}$ ) denote the common value of (13.10) (resp. (13.11)). For notational convenience define $w_{-1}=0$ and $w_{-1}^{\prime}=0$. 
Lemma 13.16. For $0 \leq i \leq N$,

$$
\begin{aligned}
\tau_{i}=\exp _{q}\left(b^{-1} \xi \psi\right) w_{i}, & w_{i}=\exp _{q^{-1}}\left(-b^{-1} \xi \psi\right) \tau_{i} \\
\eta_{i}=\exp _{q}\left(a^{-1} \xi \psi\right) w_{i}, & w_{i}=\exp _{q^{-1}}\left(-a^{-1} \xi \psi\right) \eta_{i}
\end{aligned}
$$

and

$$
\begin{aligned}
\tau_{i}=\exp _{q^{-1}}\left(-a^{-1} \xi \psi\right) w_{i}^{\prime}, & w_{i}^{\prime}=\exp _{q}\left(a^{-1} \xi \psi\right) \tau_{i} \\
\eta_{i}=\exp _{q^{-1}}\left(-b^{-1} \xi \psi\right) w_{i}^{\prime} & w_{i}^{\prime}=\exp _{q}\left(b^{-1} \xi \psi\right) \eta_{i} .
\end{aligned}
$$

In the above lines $\xi=1-a b$.

Proof. By Definition 13.15 and the comments above Lemma 13.12.

Note 13.17. Referring to Definition 13.15, the polynomials $\left\{w_{i}^{\prime}\right\}_{i=0}^{N}$ are obtained from the polynomials $\left\{w_{i}\right\}_{i=0}^{N}$ by replacing $q \mapsto q^{-1}, a \mapsto a^{-1}, b \mapsto b^{-1}$.

Example 13.18. The following (i)-(iii) hold:

(i) $w_{0}=1$;

(ii) $w_{1}$ is equal to each of

$$
\tau_{1}-(1-a b) b^{-1} \tau_{0}, \quad \eta_{1}-(1-a b) a^{-1} \eta_{0}, \quad x-a^{-1}-b^{-1} ;
$$

(iii) $w_{2}$ is equal to each of

$$
\begin{aligned}
& \tau_{2}-\left(q^{-1}+1\right)(1-a b q) b^{-1} \tau_{1}+q^{-1}(1-a b)(1-a b q) b^{-2} \tau_{0}, \\
& \eta_{2}-\left(q^{-1}+1\right)(1-a b q) a^{-1} \eta_{1}+q^{-1}(1-a b)(1-a b q) a^{-2} \eta_{0}, \\
& \left(x-a^{-1}-b^{-1}\right)\left(x-q^{-1} a^{-1}-q^{-1} b^{-1}\right)+\left(q^{-1}-1\right)\left(1-a^{-1} b^{-1}\right) .
\end{aligned}
$$

To get $w_{0}^{\prime}, w_{1}^{\prime}, w_{2}^{\prime}$ replace $q \mapsto q^{-1}, a \mapsto a^{-1}, b \mapsto b^{-1}$ in (i)-(iii) above.

Lemma 13.19. The following $(i)-($ iii $)$ hold:

(i) for $0 \leq i \leq N$ the polynomials $w_{i}$, $w_{i}^{\prime}$ are monic with degree $i$;

(ii) for $0 \leq n \leq N$, each of $\left\{w_{i}\right\}_{i=0}^{n},\left\{w_{i}^{\prime}\right\}_{i=0}^{n}$ is a basis for the vector space $V_{n}$;

(iii) each of $\left\{w_{i}\right\}_{i=0}^{N},\left\{w_{i}^{\prime}\right\}_{i=0}^{N}$ is a basis for the vector space $V$.

Proof. (i) By (4.9) and Definition 13.15. (ii), (iii) By (i) above.

Lemma 13.20. For $0 \leq j \leq N$,

$$
\begin{aligned}
w_{j}^{\prime} & =a^{-j}(a b ; q)_{j} \sum_{i=0}^{j} \frac{\left(q^{-j} ; q\right)_{i}(a y ; q)_{i}\left(a y^{-1} ; q\right)_{i} q^{i}}{(a b ; q)_{i}(q ; q)_{i}} \\
& =a^{-j}(a b ; q)_{j} \phi_{2}\left(\begin{array}{c}
q^{-j}, a y, a y^{-1} \\
a b, 0
\end{array} \mid q ; q\right)
\end{aligned}
$$

where $x=y+y^{-1}$. To get $w_{j}$ from $w_{j}^{\prime}$, replace $q \mapsto q^{-1}, a \mapsto a^{-1}, b \mapsto b^{-1}$. 
Proof. In the equation $w_{j}^{\prime}=\exp _{q}\left(a^{-1} \xi \psi\right) \tau_{j}$, expand the $q$-exponential using (13.5), and evaluate the result using the equation on the left in (4.9). This yields $w_{j}^{\prime}=\sum_{i=0}^{j} \alpha_{i} \tau_{i}$ where for $0 \leq i \leq j$

$$
\alpha_{i}=\frac{q^{\left(\frac{j-i}{2}\right)}(1-q)^{j-i} a^{i-j} \xi^{j-i} \vartheta_{j} \vartheta_{j-1} \cdots \vartheta_{i+1}}{(q ; q)_{j-i}} .
$$

Evaluating this using

$$
\vartheta_{j} \vartheta_{j-1} \cdots \vartheta_{i+1}=\frac{\vartheta_{1} \vartheta_{2} \cdots \vartheta_{j}}{\vartheta_{1} \vartheta_{2} \cdots \vartheta_{i}}
$$

and (13.2), (13.3) we obtain

$$
\alpha_{i}=\frac{(-1)^{i} a^{i-j}(a b ; q)_{j}\left(q^{-j} ; q\right)_{i} q^{\left(\begin{array}{l}
i \\
2
\end{array}\right)} q^{i}}{(a b ; q)_{i}(q ; q)_{i}} .
$$

The polynomial $\tau_{i}$ is given in Lemma 13.10(i). The result follows.

Note 13.21. The polynomials $\left\{w_{i}\right\}_{i=0}^{N}$ and $\left\{w_{i}^{\prime}\right\}_{i=0}^{N}$ are in the Al-Salam/Chihara family [25, Section 14.8] if $N=\infty$, and the dual $q$-Krawtchouk family [25, Section 14.17] if $N \neq \infty$. The Al-Salam/Chihara and dual $q$-Krawtchouk polynomials satisfy a 3-term recurrence; the details will be given in Lemmas 13.67 and 13.68 below.

Going forward we focus on $\left\{w_{i}\right\}_{i=0}^{N}$; similar results hold for $\left\{w_{i}^{\prime}\right\}_{i=0}^{N}$.

Lemma 13.22. We have

$$
\psi w_{i}=\vartheta_{i} w_{i-1}, \quad 0 \leq i \leq N .
$$

Proof. By Definition 13.15 and since $\psi \tau_{i}=\vartheta_{i} \tau_{i-1}$.

Our next general goal is to describe in more detail how the bases $\left\{\tau_{i}\right\}_{i=0}^{N},\left\{\eta_{i}\right\}_{i=0}^{N},\left\{w_{i}\right\}_{i=0}^{N}$ are related. To this end, we introduce some maps $K, B, M \in \operatorname{End}(V)$.

Definition 13.23. Define $K, B, M \in \operatorname{End}(V)$ such that for $0 \leq i \leq N$,

$$
K \tau_{i}=q^{-i} \tau_{i}, \quad B \eta_{i}=q^{-i} \eta_{i}, \quad M w_{i}=q^{-i} w_{i} .
$$

Each of $K, B, M$ is invertible.

Lemma 13.24. The following (i)-(iii) hold:

(i) $K \psi=q \psi K$;

(ii) $B \psi=q \psi B$;

(iii) $M \psi=q \psi M$.

Proof. (i) The vectors $\left\{\tau_{i}\right\}_{i=0}^{N}$ form a basis for $V$. For $0 \leq i \leq N$,

$$
K \psi \tau_{i}=\vartheta_{i} K \tau_{i-1}=\vartheta_{i} q^{1-i} \tau_{i-1}, \quad q \psi K \tau_{i}=q^{1-i} \psi \tau_{i}=\vartheta_{i} q^{1-i} \tau_{i-1}
$$

Therefore $K \psi=q \psi K$. (ii), (iii) Similar to the proof of (i) above.

Lemma 13.25. The following (i)-(iii) hold:

(i) $B \Delta=\Delta K$; 
(ii) $K \exp _{q}\left(b^{-1} \xi \psi\right)=\exp _{q}\left(b^{-1} \xi \psi\right) M$;

(iii) $B \exp _{q}\left(a^{-1} \xi \psi\right)=\exp _{q}\left(a^{-1} \xi \psi\right) M$.

Proof. (i) For $0 \leq i \leq N$, apply each side to $\tau_{i}$ and use (2.5) along with Definition 13.23.

(ii), (iii) For $0 \leq i \leq N$, apply each side to $w_{i}$ and use Lemma 13.16 along with Definition 13.23 .

Proposition 13.26. The following $(i)-(i v)$ hold:

(i) $K M^{-1}=I+(q-1)\left(a-b^{-1}\right) \psi$;

(ii) $M^{-1} K=I+\left(q^{-1}-1\right)\left(b^{-1}-a\right) \psi$;

(iii) $B M^{-1}=I+(q-1)\left(b-a^{-1}\right) \psi$;

(iv) $M^{-1} B=I+\left(q^{-1}-1\right)\left(a^{-1}-b\right) \psi$.

Proof. (i) The map $T=b^{-1} \xi \psi$ is locally nilpotent. We have $K T K^{-1}=q T$ by Lemma 13.24(i), and $K \exp _{q}(T)=\exp _{q}(T) M$ by Lemma 13.25(ii). By these comments and Lemma 13.12,

$$
\begin{aligned}
\exp _{q}(T) & =(I-(q-1) T) \exp _{q}(q T)=(I-(q-1) T) \exp _{q}\left(K T K^{-1}\right) \\
& =(I-(q-1) T) K \exp _{q}(T) K^{-1}=(I-(q-1) T) \exp _{q}(T) M K^{-1} \\
& =\exp _{q}(T)(I-(q-1) T) M K^{-1} .
\end{aligned}
$$

By this and since $\exp _{q}(T)$ is invertible,

$$
I=(I-(q-1) T) M K^{-1} .
$$

The result follows from this and $\xi=1-a b$.

(ii) By (i) above and $M \psi=q \psi M$. (iii), (iv) Similar to the proof of (i), (ii) above.

Corollary 13.27. We have

$$
\begin{aligned}
& \psi=\frac{b q}{1-a b} \frac{M^{-1} K-K M^{-1}}{(q-1)^{2}}, \\
& \psi=\frac{a q}{1-a b} \frac{M^{-1} B-B M^{-1}}{(q-1)^{2}} .
\end{aligned}
$$

Proof. To get (13.14) use Proposition 13.26(i), (ii).

To get (13.15) use Proposition 13.26(iii), (iv).

Proposition 13.28. We have

$$
M=\frac{b K-a B}{b-a} .
$$

Proof. Compute $b$ times Proposition 13.26(i) minus $a$ times Proposition 13.26(iii).

Lemma 13.29. Each of the following is invertible:

$$
a I-b B^{-1} K, \quad b I-a K^{-1} B, \quad a^{-1} I-b^{-1} B K^{-1}, \quad b^{-1} I-a^{-1} K B^{-1} .
$$

Proof. By Proposition 13.28 and since $M$ is invertible.

Our next goal is to describe how $K, B$ are related. We will use the following result. 
Lemma 13.30. We have

$$
\begin{aligned}
\psi M & =\frac{1}{1-q} \frac{a b}{1-a b} \frac{K-B}{a-b}, \\
M \psi & =\frac{q}{1-q} \frac{a b}{1-a b} \frac{K-B}{a-b} .
\end{aligned}
$$

Proof. To get (13.16), subtract Proposition 13.26(iii) from Proposition 13.26(i). To get (13.17) from (13.16), use $M \psi=q \psi M$.

Proposition 13.31. We have

$$
(b K-a B)(K-B)=q(K-B)(b K-a B) .
$$

Proof. We have $M \psi=q \psi M$ so $M(\psi M)=q(\psi M) M$. Evaluate this using Proposition 13.28 and (13.16).

We mention some reformulations of Proposition 13.31.

Corollary 13.32. We have

$$
0=a B^{2}-\frac{b q-a}{q-1} B K-\frac{a q-b}{q-1} K B+b K^{2}
$$

and

$$
\begin{aligned}
\left(b I-a K^{-1} B\right)\left(I-K B^{-1}\right) & =q\left(I-K^{-1} B\right)\left(a I-b K B^{-1}\right), \\
\left(a I-b B^{-1} K\right)\left(I-K B^{-1}\right) & =q\left(I-B^{-1} K\right)\left(a I-b K B^{-1}\right), \\
\left(a I-b B^{-1} K\right)\left(I-B K^{-1}\right) & =q\left(I-B^{-1} K\right)\left(b I-a B K^{-1}\right), \\
\left(b I-a K^{-1} B\right)\left(I-B K^{-1}\right) & =q\left(I-K^{-1} B\right)\left(b I-a B K^{-1}\right) .
\end{aligned}
$$

Proposition 13.33. We have

$$
\begin{aligned}
K B^{-1} & =\frac{I+(q-1)\left(a-b^{-1}\right) \psi}{I+(q-1)\left(b-a^{-1}\right) \psi}, \\
B K^{-1} & =\frac{I+(q-1)\left(b-a^{-1}\right) \psi}{I+(q-1)\left(a-b^{-1}\right) \psi}, \\
K^{-1} B & =\frac{I+\left(q^{-1}-1\right)\left(a^{-1}-b\right) \psi}{I+\left(q^{-1}-1\right)\left(b^{-1}-a\right) \psi}, \\
B^{-1} K & =\frac{I+\left(q^{-1}-1\right)\left(b^{-1}-a\right) \psi}{I+\left(q^{-1}-1\right)\left(a^{-1}-b\right) \psi} .
\end{aligned}
$$

In the above fractions the denominator is invertible since $\psi$ is locally nilpotent.

Proof. To get (13.18), equate the two expressions for $M^{-1}$ obtained from Proposition 13.26(i) and (iii). To get (13.19) from (13.18), compute the inverse of each side. To get (13.20), equate the two expressions for $M^{-1}$ obtained from Proposition 13.26(ii) and (iv). To get (13.21) from (13.20), compute the inverse of each side.

Lemma 13.34. The following mutually commute:

$$
\psi, \quad K B^{-1}, \quad B K^{-1}, \quad K^{-1} B, \quad B^{-1} K .
$$


Proof. By Proposition 13.33.

Proposition 13.35. We have

$$
\begin{aligned}
& \psi=\frac{1}{q-1} \frac{1}{1-a b} \frac{I-K B^{-1}}{b^{-1} I-a^{-1} K B^{-1}}, \\
& \psi=\frac{1}{q-1} \frac{1}{1-a b} \frac{I-B K^{-1}}{a^{-1} I-b^{-1} B K^{-1}}, \\
& \psi=\frac{1}{q^{-1}-1} \frac{1}{1-a^{-1} b^{-1}} \frac{I-K^{-1} B}{b I-a K^{-1} B}, \\
& \psi=\frac{1}{q^{-1}-1} \frac{1}{1-a^{-1} b^{-1}} \frac{I-B^{-1} K}{a I-b B^{-1} K} .
\end{aligned}
$$

In the above fractions the denominator is invertible by Lemma 13.29.

Proof. In Proposition 13.33 solve for $\psi$.

Proposition 13.36. We have

$$
\begin{aligned}
& \frac{q M^{-1} K-K M^{-1}}{q-1}=I, \\
& \frac{q M^{-1} B-B M^{-1}}{q-1}=I .
\end{aligned}
$$

Proof. To get (13.22) use Proposition 13.26(i) and (ii). To get (13.23) use Proposition 13.26(iii) and (iv).

We have a comment.

Lemma 13.37. The relations in Proposition 13.31 and Corollary 13.32 still hold if we replace

$$
q \mapsto q^{-1}, \quad a \mapsto a^{-1}, \quad b \mapsto b^{-1}, \quad K \mapsto K^{-1}, \quad B \mapsto B^{-1} .
$$

Proof. Use Proposition 13.31 and Lemma 13.34.

We recall some notation. Let $\operatorname{Mat}_{N+1}(\mathbb{F})$ denote the set of $N+1$ by $N+1$ matrices that have all entries in $\mathbb{F}$. We index the rows and columns by $0,1, \ldots, N$. Let $\left\{v_{i}\right\}_{i=0}^{N}$ denote a basis for $V$. For $M \in \operatorname{Mat}_{N+1}(\mathbb{F})$ and $T \in \operatorname{End}(V)$, we say that $M$ represents $T$ with respect to $\left\{v_{i}\right\}_{i=0}^{N}$ whenever $T v_{j}=\sum_{i=0}^{N} M_{i, j} v_{i}$ for $0 \leq j \leq N$.

Our next goal is to display the matrices that represent $\psi, K^{ \pm 1}, M^{ \pm 1}, B^{ \pm 1}$ with respect to the bases $\left\{\tau_{i}\right\}_{i=0}^{N},\left\{w_{i}\right\}_{i=0}^{N},\left\{\eta_{i}\right\}_{i=0}^{N}$ of $V$.

Definition 13.38. Let $\widehat{\psi}$ denote the matrix in $\operatorname{Mat}_{N+1}(\mathbb{F})$ that has $(i-1, i)$-entry $\vartheta_{i}$ for $1 \leq$ $i \leq N$, and all other entries 0 . Thus

$$
\widehat{\psi}=\left(\begin{array}{cccccc}
0 & \vartheta_{1} & & & & \mathbf{0} \\
& 0 & \vartheta_{2} & & & \\
& & 0 & \cdot & & \\
& & & \cdot & \cdot & \\
& & & & \cdot & \vartheta_{N} \\
\mathbf{0} & & & & & 0
\end{array}\right) .
$$

Lemma 13.39. The matrix $\widehat{\psi}$ represents $\psi$ with respect to $\left\{\tau_{i}\right\}_{i=0}^{N}$ and $\left\{w_{i}\right\}_{i=0}^{N}$ and $\left\{\eta_{i}\right\}_{i=0}^{N}$. 
Proof. By (4.9) and Lemma 13.22.

Lemma 13.40. The matrix $\operatorname{diag}\left(1, q^{-1}, q^{-2}, \ldots, q^{-N}\right)$ represents $K$ (resp. $\left.M\right)$ (resp. B) with respect to $\left\{\tau_{i}\right\}_{i=0}^{N}$ (resp. $\left.\left\{w_{i}\right\}_{i=0}^{N}\right)$ (resp. $\left.\left\{\eta_{i}\right\}_{i=0}^{N}\right)$.

Proof. By Definition 13.23.

Lemma 13.41. We give the matrix in $\mathrm{Mat}_{N+1}(\mathbb{F})$ that represents $K$ with respect to $\left\{w_{i}\right\}_{i=0}^{N}$. The $(i, i)$-entry is $q^{-i}$ for $0 \leq i \leq N$. The $(i-1, i)$-entry is $\left(1-q^{-i}\right)\left(a-b^{-1} q^{1-i}\right)$ for $1 \leq i \leq N$. All other entries are 0.

Proof. Use $K M^{-1}=I+(q-1)\left(a-b^{-1}\right) \psi$ and Lemmas 13.6(ii), 13.39, 13.40.

Lemma 13.42. We give the matrix in $\operatorname{Mat}_{N+1}(\mathbb{F})$ that represents $B$ with respect to $\left\{w_{i}\right\}_{i=0}^{N}$. The $(i, i)$-entry is $q^{-i}$ for $0 \leq i \leq N$. The $(i-1, i)$-entry is $\left(1-q^{-i}\right)\left(b-a^{-1} q^{1-i}\right)$ for $1 \leq i \leq N$. All other entries are 0.

Proof. Use $B M^{-1}=I+(q-1)\left(b-a^{-1}\right) \psi$ and Lemmas 13.6(ii), 13.39, 13.40.

Lemma 13.43. We give the matrix in $\mathrm{Mat}_{N+1}(\mathbb{F})$ that represents $M^{-1}$ with respect to $\left\{\tau_{i}\right\}_{i=0}^{N}$. The $(i, i)$-entry is $q^{i}$ for $0 \leq i \leq N$. The $(i-1, i)$-entry is $\left(q^{i}-1\right)\left(a q^{i-1}-b^{-1}\right)$ for $1 \leq i \leq N$. All other entries are 0.

Proof. Use $M^{-1} K=I+\left(q^{-1}-1\right)\left(b^{-1}-a\right) \psi$ and Lemmas 13.6(i), 13.39, 13.40.

Lemma 13.44. We give the matrix in $\mathrm{Mat}_{N+1}(\mathbb{F})$ that represents $M^{-1}$ with respect to $\left\{\eta_{i}\right\}_{i=0}^{N}$. The $(i, i)$-entry is $q^{i}$ for $0 \leq i \leq N$. The $(i-1, i)$-entry is $\left(q^{i}-1\right)\left(b q^{i-1}-a^{-1}\right)$ for $1 \leq i \leq N$. All other entries are 0.

Proof. Use $M^{-1} B=I+\left(q^{-1}-1\right)\left(a^{-1}-b\right) \psi$ and Lemmas 13.6(i), 13.39, 13.40.

We give a variation on Proposition 13.26.

Lemma 13.45. We have

$$
\begin{aligned}
& M K^{-1}=\sum_{i=0}^{N}(-1)^{i}(1-q)^{i}(1-a b)^{i} b^{-i} \psi^{i}, \\
& K^{-1} M=\sum_{i=0}^{N}(-1)^{i}(1-q)^{i} q^{-i}(1-a b)^{i} b^{-i} \psi^{i}, \\
& M B^{-1}=\sum_{i=0}^{N}(-1)^{i}(1-q)^{i}(1-a b)^{i} a^{-i} \psi^{i}, \\
& B^{-1} M=\sum_{i=0}^{N}(-1)^{i}(1-q)^{i} q^{-i}(1-a b)^{i} a^{-i} \psi^{i} .
\end{aligned}
$$

Proof. For each equation in Proposition 13.26, take the inverse of each side and evaluate the result using Lemma 2.14 .

Lemma 13.46. We give the matrix in $\operatorname{Mat}_{N+1}(\mathbb{F})$ that represents $M$ with respect to $\left\{\tau_{i}\right\}_{i=0}^{N}$. The $(i, j)$-entry is

$$
\frac{(-1)^{j-i} b^{i-j}(a b ; q)_{j}(q ; q)_{j} q^{\left(\begin{array}{c}
i \\
2
\end{array}\right)-\left(\begin{array}{c}
j \\
2
\end{array}\right)-j}}{(a b ; q)_{i}(q ; q)_{i}}
$$

for $0 \leq i \leq j \leq N$. All other entries are 0 . 
Proof. Use (13.3), (13.24) and Lemmas 13.39, 13.40.

Lemma 13.47. We give the matrix in $\operatorname{Mat}_{N+1}(\mathbb{F})$ that represents $K^{-1}$ with respect to $\left\{w_{i}\right\}_{i=0}^{N}$. The $(i, j)$-entry is

$$
\frac{(-1)^{j-i} b^{i-j}(a b ; q)_{j}(q ; q)_{j} q^{\left(\begin{array}{c}
i \\
2
\end{array}\right)-\left(\begin{array}{c}
j \\
2
\end{array}\right)+i}}{(a b ; q)_{i}(q ; q)_{i}}
$$

for $0 \leq i \leq j \leq N$. All other entries are 0 .

Proof. Use (13.3), (13.25) and Lemmas 13.39, 13.40.

Lemma 13.48. We give the matrix in $\operatorname{Mat}_{N+1}(\mathbb{F})$ that represents $M$ with respect to $\left\{\eta_{i}\right\}_{i=0}^{N}$. The $(i, j)$-entry is

$$
\frac{(-1)^{j-i} a^{i-j}(a b ; q)_{j}(q ; q)_{j} q^{\left(\begin{array}{c}
i \\
2
\end{array}\right)-\left(\begin{array}{c}
j \\
2
\end{array}\right)-j}}{(a b ; q)_{i}(q ; q)_{i}}
$$

for $0 \leq i \leq j \leq N$. All other entries are 0 .

Proof. Use (13.3), (13.26) and Lemmas 13.39, 13.40.

Lemma 13.49. We give the matrix in $\operatorname{Mat}_{N+1}(\mathbb{F})$ that represents $B^{-1}$ with respect to $\left\{w_{i}\right\}_{i=0}^{N}$. The $(i, j)$-entry is

$$
\frac{(-1)^{j-i} a^{i-j}(a b ; q)_{j}(q ; q)_{j} q^{\left(\begin{array}{c}
i \\
2
\end{array}\right)-\left(\begin{array}{c}
j \\
2
\end{array}\right)+i}}{(a b ; q)_{i}(q ; q)_{i}}
$$

for $0 \leq i \leq j \leq N$. All other entries are 0 .

Proof. Use (13.3), (13.27) and Lemmas 13.39, 13.40.

We give a variation on Proposition 13.33.

Lemma 13.50. We have

$$
\begin{aligned}
& K B^{-1}=I+\frac{b-a}{b} \sum_{i=1}^{N}(-1)^{i}(1-q)^{i}(1-a b)^{i} a^{-i} \psi^{i}, \\
& B K^{-1}=I+\frac{a-b}{a} \sum_{i=1}^{N}(-1)^{i}(1-q)^{i}(1-a b)^{i} b^{-i} \psi^{i}, \\
& K^{-1} B=I+\frac{a-b}{a} \sum_{i=1}^{N}(-1)^{i}(1-q)^{i} q^{-i}(1-a b)^{i} b^{-i} \psi^{i}, \\
& B^{-1} K=I+\frac{b-a}{b} \sum_{i=1}^{N}(-1)^{i}(1-q)^{i} q^{-i}(1-a b)^{i} a^{-i} \psi^{i} .
\end{aligned}
$$

Proof. Evaluate each equation in Proposition 13.33 using Lemma 2.14.

Lemma 13.51. We give the matrix in $\operatorname{Mat}_{N+1}(\mathbb{F})$ that represents $K$ with respect to $\left\{\eta_{i}\right\}_{i=0}^{N}$. The $(i, i)$-entry is $q^{-i}$ for $0 \leq i \leq N$. The $(i, j)$-entry is

$$
\frac{b-a}{b} \frac{(-1)^{j-i} a^{i-j}(a b ; q)_{j}(q ; q)_{j} q^{\left(\begin{array}{c}
i \\
2
\end{array}\right)-\left(\begin{array}{c}
j \\
2
\end{array}\right)-j}}{(a b ; q)_{i}(q ; q)_{i}}
$$

for $0 \leq i<j \leq N$. All other entries are 0 . 
Proof. Use (13.3), (13.28) and Lemmas 13.39, 13.40.

Lemma 13.52. We give the matrix in $\operatorname{Mat}_{N+1}(\mathbb{F})$ that represents $B$ with respect to $\left\{\tau_{i}\right\}_{i=0}^{N}$. The $(i, i)$-entry is $q^{-i}$ for $0 \leq i \leq N$. The $(i, j)$-entry is

$$
\frac{a-b}{a} \frac{(-1)^{j-i} b^{i-j}(a b ; q)_{j}(q ; q)_{j} q^{\left(\begin{array}{c}
i \\
2
\end{array}\right)-\left(\begin{array}{c}
j \\
2
\end{array}\right)-j}}{(a b ; q)_{i}(q ; q)_{i}}
$$

for $0 \leq i<j \leq N$. All other entries are 0 .

Proof. Use (13.3), (13.29) and Lemmas 13.39, 13.40.

Lemma 13.53. We give the matrix in $\operatorname{Mat}_{N+1}(\mathbb{F})$ that represents $K^{-1}$ with respect to $\left\{\eta_{i}\right\}_{i=0}^{N}$. The $(i, i)$-entry is $q^{i}$ for $0 \leq i \leq N$. The $(i, j)$-entry is

$$
\frac{a-b}{a} \frac{(-1)^{j-i} b^{i-j}(a b ; q)_{j}(q ; q)_{j} q^{\left(\begin{array}{c}
i \\
2
\end{array}\right)-\left(\begin{array}{c}
j \\
2
\end{array}\right)+i}}{(a b ; q)_{i}(q ; q)_{i}}
$$

for $0 \leq i<j \leq N$. All other entries are 0 .

Proof. Use (13.3), (13.30) and Lemmas 13.39, 13.40.

Lemma 13.54. We give the matrix in $\operatorname{Mat}_{N+1}(\mathbb{F})$ that represents $B^{-1}$ with respect to $\left\{\tau_{i}\right\}_{i=0}^{N}$. The $(i, i)$-entry is $q^{i}$ for $0 \leq i \leq N$. The $(i, j)$-entry is

$$
\frac{b-a}{b} \frac{(-1)^{j-i} a^{i-j}(a b ; q)_{j}(q ; q)_{j} q^{\left(\begin{array}{c}
i \\
2
\end{array}\right)-\left(\begin{array}{c}
j \\
2
\end{array}\right)+i}}{(a b ; q)_{i}(q ; q)_{i}}
$$

for $0 \leq i<j \leq N$. All other entries are 0 .

Proof. Use (13.3), (13.31) and Lemmas 13.39, 13.40.

We recall some notation. Let $\left\{u_{i}\right\}_{i=0}^{N}$ and $\left\{v_{i}\right\}_{i=0}^{N}$ denote bases for $V$. By the transition matrix from $\left\{u_{i}\right\}_{i=0}^{N}$ to $\left\{v_{i}\right\}_{i=0}^{N}$ we mean the matrix $T \in \operatorname{Mat}_{N+1}(\mathbb{F})$ such that $v_{j}=\sum_{i=0}^{N} T_{i, j} u_{i}$ for $0 \leq j \leq N$.

Our next goal is to display the transition matrices between the bases $\left\{\tau_{i}\right\}_{i=0}^{N},\left\{w_{i}\right\}_{i=0}^{N},\left\{\eta_{i}\right\}_{i=0}^{N}$. Recall the notation $\xi=1-a b$.

Consider the following matrices:

$$
\exp _{q}\left(a^{-1} \xi \widehat{\psi}\right), \quad \exp _{q}\left(b^{-1} \xi \widehat{\psi}\right) .
$$

Their inverses are

$$
\exp _{q^{-1}}\left(-a^{-1} \xi \widehat{\psi}\right), \quad \exp _{q^{-1}}\left(-b^{-1} \xi \widehat{\psi}\right) .
$$

The matrices (13.32), (13.33) are upper triangular. We now give their entries.

Lemma 13.55. For $0 \neq z \in \mathbb{F}$ the matrix $\exp _{q}(z \xi \widehat{\psi})$ is upper triangular. Its $(i, j)$-entry is

$$
\frac{(-1)^{i} z^{j-i}(a b ; q)_{j}\left(q^{-j} ; q\right)_{i} q^{i+\left(\begin{array}{c}
i \\
2
\end{array}\right)}}{(a b ; q)_{i}(q ; q)_{i}}
$$

for $0 \leq i \leq j \leq N$. The matrix $\exp _{q^{-1}}(-z \xi \widehat{\psi})$ is upper triangular. Its $(i, j)$-entry is

$$
\frac{(-1)^{j} z^{j-i}(a b ; q)_{j}\left(q^{-j} ; q\right)_{i} q^{i j-\left(\begin{array}{c}
j \\
2
\end{array}\right)}}{(a b ; q)_{i}(q ; q)_{i}}
$$

for $0 \leq i \leq j \leq N$. 
Proof. Use (13.2), (13.3), (13.5), (13.6) and Definition 13.38.

Lemma 13.56. The transition matrices between the basis $\left\{w_{i}\right\}_{i=0}^{N}$ and the bases $\left\{\tau_{i}\right\}_{i=0}^{N},\left\{\eta_{i}\right\}_{i=0}^{N}$ are given in the table below:

\begin{tabular}{ccc} 
from & to & transition matrix \\
\hline$\left\{\tau_{i}\right\}_{i=0}^{N}$ & $\left\{w_{i}\right\}_{i=0}^{N}$ & $\exp _{q^{-1}}\left(-b^{-1} \xi \widehat{\psi}\right)$ \\
$\left\{w_{i}\right\}_{i=0}^{N}$ & $\left\{\tau_{i}\right\}_{i=0}^{N}$ & $\exp _{q}\left(b^{-1} \xi \widehat{\psi}\right)$ \\
$\left\{\eta_{i}\right\}_{i=0}^{N}$ & $\left\{w_{i}\right\}_{i=0}^{N}$ & $\exp _{q^{-1}}\left(-a^{-1} \xi \widehat{\psi}\right)$ \\
$\left\{w_{i}\right\}_{i=0}^{N}$ & $\left\{\eta_{i}\right\}_{i=0}^{N}$ & $\exp _{q}\left(a^{-1} \xi \widehat{\psi}\right)$
\end{tabular}

Proof. By Lemmas 13.16, 13.39.

Next we consider the product

$$
\exp _{q}\left(a^{-1} \xi \widehat{\psi}\right) \exp _{q^{-1}}\left(-b^{-1} \xi \widehat{\psi}\right)
$$

The inverse of (13.34) is

$$
\exp _{q}\left(b^{-1} \xi \widehat{\psi}\right) \exp _{q^{-1}}\left(-a^{-1} \xi \widehat{\psi}\right) \text {. }
$$

The matrices (13.34), (13.35) are upper triangular. Shortly we will give their entries.

Lemma 13.57. The matrix (13.34) is the transition matrix from the basis $\left\{\tau_{i}\right\}_{i=0}^{N}$ to the basis $\left\{\eta_{i}\right\}_{i=0}^{N}$. The matrix (13.35) is the transition matrix from the basis $\left\{\eta_{i}\right\}_{i=0}^{N}$ to the basis $\left\{\tau_{i}\right\}_{i=0}^{N}$.

Proof. By Lemma 13.56.

Lemma 13.58. The matrix (13.34) represents $\Delta$ with respect to $\left\{\tau_{i}\right\}_{i=0}^{N}$ and $\left\{w_{i}\right\}_{i=0}^{N}$ and $\left\{\eta_{i}\right\}_{i=0}^{N}$. The matrix (13.35) represents $\Delta^{-1}$ with respect to $\left\{\tau_{i}\right\}_{i=0}^{N}$ and $\left\{w_{i}\right\}_{i=0}^{N}$ and $\left\{\eta_{i}\right\}_{i=0}^{N}$.

Proof. By Proposition 13.13 and Lemma 13.39.

Lemma 13.59. The matrix (13.34) is upper triangular, with $(i, j)$-entry

$$
\eta_{j-i}\left(a_{0}\right)\left[\begin{array}{l}
j \\
i
\end{array}\right]_{\vartheta}
$$

for $0 \leq i \leq j \leq N$. The matrix (13.35) is upper triangular, with $(i, j)$-entry

$$
\tau_{j-i}\left(b_{0}\right)\left[\begin{array}{l}
j \\
i
\end{array}\right]_{\vartheta}
$$

for $0 \leq i \leq j \leq N$. To express (13.36), (13.37) in terms of $a, b, q$ use Lemmas 13.9, 13.11.

Proof. By Lemma 13.57, the matrix (13.34) is the transition matrix from the basis $\left\{\tau_{i}\right\}_{i=0}^{N}$ to the basis $\left\{\eta_{i}\right\}_{i=0}^{N}$. The entries of this matrix are obtained from Proposition 5.2(ii). The entries of the matrix (13.35) are similarly obtained.

Our next goal is to show how the map $A$ from Definition 2.11 is related to $\psi, K, B, M$.

Lemma 13.60. On $V_{N-1}$,

$$
\begin{aligned}
& \frac{q K A-A K}{q-1}=a^{-1} K^{2}+a I, \\
& \frac{q B A-A B}{q-1}=b^{-1} B^{2}+b I .
\end{aligned}
$$


Proof. We first show (13.38). For $0 \leq i \leq N-1$ apply each side of (13.38) to $\tau_{i}$, and evaluate the result using Lemma 2.13 along with (13.1) and Definition 13.23. We have

$$
\begin{aligned}
& K A \tau_{i}=K\left(a_{i} \tau_{i}+\tau_{i+1}\right)=q^{-i} a_{i} \tau_{i}+q^{-i-1} \tau_{i+1}, \\
& A K \tau_{i}=q^{-i} A \tau_{i}=q^{-i}\left(a_{i} \tau_{i}+\tau_{i+1}\right), \\
& \left(a^{-1} K^{2}+a I\right) \tau_{i}=\left(a^{-1} q^{-2 i}+a\right) \tau_{i}=q^{-i} a_{i} \tau_{i} .
\end{aligned}
$$

By these comments we obtain (13.38). Equation (13.39) is similarly obtained.

Lemma 13.61. On $V_{N-1}$,

$$
q \psi A-A \psi=\frac{(q+1) a b M^{-1}-(q+a b) I}{a b-1} .
$$

Proof. For $0 \leq i \leq N-1$ apply each side of (13.40) to $\tau_{i}$. Using Lemma 2.13 and (4.9),

$$
\begin{aligned}
& \psi A \tau_{i}=\psi\left(a_{i} \tau_{i}+\tau_{i+1}\right)=a_{i} \vartheta_{i} \tau_{i-1}+\vartheta_{i+1} \tau_{i} \\
& A \psi \tau_{i}=\vartheta_{i} A \tau_{i-1}=\vartheta_{i}\left(a_{i-1} \tau_{i-1}+\tau_{i}\right) .
\end{aligned}
$$

Using Lemma 13.6(i) and Lemma 13.43,

$$
M^{-1} \tau_{i}=q^{i} \tau_{i}+(q-1)\left(a-b^{-1}\right) q^{i-1} \vartheta_{i} \tau_{i-1} .
$$

By the above comments and Lemmas 13.4, 13.7 we get the result.

Lemma 13.62. On $V_{N-1}$,

$$
\frac{q A M^{-1}-M^{-1} A}{q-1}=\left(a^{-1}+b^{-1}\right) I+\left(q-q^{-1}\right)\left(1-a^{-1} b^{-1}\right) \psi .
$$

Proof. For $0 \leq i \leq N-1$ apply each side of (13.41) to $\tau_{i}$. Evaluate the result using (4.9) and Lemmas 2.13, 13.43 along with Lemmas 13.4, 13.7.

Proposition 13.63. On $V_{N-2}$,

$$
A^{2} \psi-\left(q+q^{-1}\right) A \psi A+\psi A^{2}+\left(q-q^{-1}\right)^{2} \psi=\frac{(1-q)\left(1+q^{-1} a b\right)}{1-a b} A+\frac{\left(q-q^{-1}\right)(a+b)}{1-a b} I .
$$

Proof. Let $X$ denote the expression on either side of (13.40). Compute

$$
\frac{q A X-X A}{q-1}
$$

and evaluate the result using Lemma 13.62.

Proposition 13.64. On $V_{N-1}$,

$$
\psi^{2} A-\left(q+q^{-1}\right) \psi A \psi+A \psi^{2}=\frac{(1-q)\left(1+q^{-1} a b\right)}{1-a b} \psi
$$

Proof. Let $X$ denote the expression on either side of (13.40). Compute $q X \psi-\psi X$ and evaluate the result using $q M^{-1} \psi=\psi M^{-1}$.

Proposition 13.65. On $V_{N-2}$,

$$
\begin{aligned}
& A^{2} M^{-1}-\left(q+q^{-1}\right) A M^{-1} A+M^{-1} A^{2}+\left(q-q^{-1}\right)^{2} M^{-1} \\
& \quad=(q-1)\left(q-q^{-1}\right)\left(q^{-1}+a^{-1} b^{-1}\right) I-q^{-1}(q-1)^{2}\left(a^{-1}+b^{-1}\right) A .
\end{aligned}
$$


Proof. Let $Y$ denote the expression on either side of (13.41). Compute $q Y A-A Y$ and evaluate the result using Lemma 13.61.

Proposition 13.66. On $V_{N-1}$,

$$
M^{-2} A-\left(q+q^{-1}\right) M^{-1} A M^{-1}+A M^{-2}=(q-1)\left(q^{-1}-1\right)\left(a^{-1}+b^{-1}\right) M^{-1} .
$$

Proof. Let $Y$ denote the expression on either side of (13.41). Compute $q M^{-1} Y-Y M^{-1}$ and evaluate the result using $q M^{-1} \psi=\psi M^{-1}$.

In Note 13.21 we mentioned a 3 -term recurrence satisfied by the polynomials $\left\{w_{i}\right\}_{i=0}^{N}$ and $\left\{w_{i}^{\prime}\right\}_{i=0}^{N}$. Our next goal is to describe this recurrence.

Lemma 13.67. We have

$$
x w_{i}=w_{i+1}+q^{-i}\left(a^{-1}+b^{-1}\right) w_{i}+\left(1-q^{-i}\right)\left(1-q^{1-i} a^{-1} b^{-1}\right) w_{i-1}
$$

for $0 \leq i \leq N-1$, where $w_{0}=1$ and $w_{-1}=0$.

Proof. The result holds for $i=0$, since $w_{1}=x-a^{-1}-b^{-1}$ by Example 13.18(ii). Assume that $i \geq 1$. By Lemma 13.19(i) there exist scalars $\left\{\alpha_{k}\right\}_{k=0}^{i+1}$ in $\mathbb{F}$ such that $x w_{i}=\sum_{k=0}^{i+1} \alpha_{k} w_{k}$ and $\alpha_{k+1}=1$. To obtain $\left\{\alpha_{k}\right\}_{k=0}^{i}$, apply each side of (13.41) to $w_{i}$ and evaluate the result using Lemma 13.22 along with $M^{-1} w_{j}=q^{j} w_{j}$ for $0 \leq j \leq i$. After a brief calculation this yields $\alpha_{i}=q^{-i}\left(a^{-1}+b^{-1}\right)$ and $\alpha_{i-1}=\left(1-q^{-i}\right)\left(1-q^{1-i} a^{-1} b^{-1}\right)$ and $\alpha_{k}=0$ for $0 \leq k \leq i-2$. The result follows.

Lemma 13.68. We have

$$
x w_{i}^{\prime}=w_{i+1}^{\prime}+q^{i}(a+b) w_{i}^{\prime}+\left(1-q^{i}\right)\left(1-q^{i-1} a b\right) w_{i-1}^{\prime}
$$

$0 \leq i \leq N-1$, where $w_{0}^{\prime}=1$ and $w_{-1}^{\prime}=0$.

Proof. In Proposition 13.67 replace $q \mapsto q^{-1}, a \mapsto a^{-1}, b \mapsto b^{-1}$ and use Note 13.17.

This completes our description of $\mathcal{L}$ and $\Delta$ for the twin recurrent data from Case I of Lemma 10.2. In this description we encountered analogs of the results from Section 1 about the double lowering operator of a tridiagonal pair. This connection suggests that double lowering operators on polynomials can be used to further develop the theory of tridiagonal pairs; we hope to pursue this in the future.

\section{Acknowledgement}

The author would like to thank Kazumasa Nomura for giving this paper a close reading and offering many valuable comments.

\section{References}

[1] Alnajjar H., Leonard pairs associated with the equitable generators of the quantum algebra $U_{q}\left(\mathfrak{s l}_{2}\right)$, Linear Multilinear Algebra 59 (2011), 1127-1142.

[2] Alnajjar H., Curtin B., A family of tridiagonal pairs related to the quantum affine algebra $\left.U_{q}(\widehat{\mathfrak{s l}})_{2}\right)$ Electron. J. Linear Algebra 13 (2005), 1-9.

[3] Askey R., Wilson J., A set of orthogonal polynomials that generalize the Racah coefficients or $6-j$ symbols, SIAM J. Math. Anal. 10 (1979), 1008-1016.

[4] Bannai E., Ito T., Algebraic combinatorics. I. Association schemes, The Benjamin/Cummings Publishing Co., Inc., Menlo Park, CA, 1984. 
[5] Baseilhac P., An integrable structure related with tridiagonal algebras, Nuclear Phys. B 705 (2005), 605619, arXiv:math-ph/0408025.

[6] Baseilhac P., The $q$-deformed analogue of the Onsager algebra: beyond the Bethe ansatz approach, Nuclear Phys. B 754 (2006), 309-328, arXiv:math-ph/0604036.

[7] Bockting-Conrad S., Two commuting operators associated with a tridiagonal pair, Linear Algebra Appl. 437 (2012), 242-270, arXiv:1110.3434.

[8] Bockting-Conrad S., Tridiagonal pairs of $q$-Racah type, the double lowering operator $\psi$, and the quantum algebra $U_{q}\left(\mathfrak{s l}_{2}\right)$, Linear Algebra Appl. 445 (2014), 256-279, arXiv:1307.7410.

[9] Bockting-Conrad S., Some $q$-exponential formulas involving the double lowering operator $\psi$ for a tridiagonal pair, arXiv:1907.01157.

[10] Bockting-Conrad S., Terwilliger P., The algebra $U_{q}\left(\mathfrak{s l}_{2}\right)$ in disguise, Linear Algebra Appl. 459 (2014), 548585, arXiv:1307.7572.

[11] Brouwer A.E., Cohen A.M., Neumaier A., Distance-regular graphs, Ergebnisse der Mathematik und ihrer Grenzgebiete (3), Vol. 18, Springer-Verlag, Berlin, 1989.

[12] Date E., Roan S., The structure of quotients of the Onsager algebra by closed ideals, J. Phys. A: Math. Gen. 33 (2000), 3275-3296, arXiv:math.QA/9911018.

[13] Gasper G., Rahman M., Basic hypergeometric series, Encyclopedia of Mathematics and its Applications, Vol. 35, Cambridge University Press, Cambridge, 1990.

[14] Granovskii Ya.I., Lutzenko I.M., Zhedanov A.S., Mutual integrability, quadratic algebras, and dynamical symmetry, Ann. Physics 217 (1992), 1-20.

[15] Hartwig B., The tetrahedron algebra and its finite-dimensional irreducible modules, Linear Algebra Appl. 422 (2007), 219-235, arXiv:math.RT/0606197.

[16] Hartwig B., Terwilliger P., The tetrahedron algebra, the Onsager algebra, and the $\mathfrak{s l}_{2}$ loop algebra, J. Algebra 308 (2007), 840-863, arXiv:math-ph/0511004.

[17] Ito T., Tanabe K., Terwilliger P., Some algebra related to $P$ - and $Q$-polynomial association schemes, in Codes and Association Schemes (Piscataway, NJ, 1999), DIMACS Ser. Discrete Math. Theoret. Comput. Sci., Vol. 56, Amer. Math. Soc., Providence, RI, 2001, 167-192, arXiv:math.CO/0406556.

[18] Ito T., Terwilliger P., Tridiagonal pairs and the quantum affine algebra $U_{q}\left(\widehat{\mathfrak{s l}}_{2}\right)$, Ramanujan J. 13 (2007), 39-62, arXiv:math.QA/0310042.

[19] Ito T., Terwilliger P., Tridiagonal pairs of Krawtchouk type, Linear Algebra Appl. 427 (2007), 218-233, arXiv:0706.1065.

[20] Ito T., Terwilliger P., Two non-nilpotent linear transformations that satisfy the cubic $q$-Serre relations, J. Algebra Appl. 6 (2007), 477-503, arXiv:math.QA/0508398.

[21] Ito T., Terwilliger P., Finite-dimensional irreducible modules for the three-point $\mathfrak{s l}_{2}$ loop algebra, Comm. Algebra 36 (2008), 4557-4598, arXiv:0707.2313.

[22] Ito T., Terwilliger P., Tridiagonal pairs of $q$-Racah type, J. Algebra 322 (2009), 68-93, arXiv:0807.0271.

[23] Ito T., Terwilliger P., The augmented tridiagonal algebra, Kyushu J. Math. 64 (2010), 81-144, arXiv:0904.2889.

[24] Ito T., Terwilliger P., Weng C., The quantum algebra $U_{q}\left(\mathfrak{s l}_{2}\right)$ and its equitable presentation, J. Algebra 298 (2006), 284-301, arXiv:math.QA/0507477.

[25] Koekoek R., Lesky P.A., Swarttouw R.F., Hypergeometric orthogonal polynomials and their $q$-analogues, Springer Monographs in Mathematics, Springer-Verlag, Berlin, 2010.

[26] Miki K., Finite dimensional modules for the q-tetrahedron algebra, Osaka J. Math. 47 (2010), 559-589.

[27] Nomura K., Terwilliger P., Krawtchouk polynomials, the Lie algebra $\mathfrak{s l}_{2}$, and Leonard pairs, Linear Algebra Appl. 437 (2012), 345-375, arXiv:1201.1645.

[28] Nomura K., Terwilliger P., Totally bipartite tridiagonal pairs, arXiv:1711.00332.

[29] Terwilliger P., The subconstituent algebra of an association scheme. I, J. Algebraic Combin. 1 (1992), 363388.

[30] Terwilliger P., Two linear transformations each tridiagonal with respect to an eigenbasis of the other, Linear Algebra Appl. 330 (2001), 149-203, arXiv:math.RA/0406555. 
[31] Terwilliger P., Two relations that generalize the $q$-Serre relations and the Dolan-Grady relations, in Physics and Combinatorics 1999 (Nagoya), World Sci. Publ., River Edge, NJ, 2001, 377-398, arXiv:math.QA/0307016.

[32] Terwilliger P., Leonard pairs and the q-Racah polynomials, Linear Algebra Appl. 387 (2004), 235-276, arXiv:math.QA/0306301.

[33] Terwilliger P., Two linear transformations each tridiagonal with respect to an eigenbasis of the other; comments on the parameter array, Des. Codes Cryptogr. 34 (2005), 307-332, arXiv:math.RA/0306291.

[34] Terwilliger P., An algebraic approach to the Askey scheme of orthogonal polynomials, in Orthogonal Polynomials and Special Functions, Lecture Notes in Math., Vol. 1883, Springer, Berlin, 2006, 255-330, arXiv:math.QA/0408390.

[35] Terwilliger P., The universal Askey-Wilson algebra, SIGMA 7 (2011), 069, 24 pages, arXiv:1104.2813.

[36] Terwilliger P., The universal Askey-Wilson algebra and the equitable presentation of $U_{q}\left(\mathfrak{s l}_{2}\right), S I G M A \mathbf{7}$ (2011), 099, 26 pages, arXiv:1107.3544.

[37] Terwilliger P., The $q$-Onsager algebra and the positive part of $U_{q}\left(\widehat{\mathfrak{s l}}_{2}\right)$, Linear Algebra Appl. 521 (2017), 19-56, arXiv:1506.08666.

[38] Terwilliger P., The $q$-Onsager algebra and the universal Askey-Wilson algebra, SIGMA 14 (2018), 044, 18 pages, arXiv:1801.06083.

[39] Terwilliger P., Tridiagonal pairs of $q$-Racah type, the Bockting operator $\psi$, and $L$-operators for $U_{q}\left(L\left(\mathfrak{s l}_{2}\right)\right)$, Ars Math. Contemp. 14 (2018), 55-65, arXiv:1608.07613.

[40] Terwilliger P., Vidunas R., Leonard pairs and the Askey-Wilson relations, J. Algebra Appl. 3 (2004), 411426, arXiv:math.QA/0305356.

[41] Vidunas R., Simultaneously lowering operators, RIMS Kōkyūroku 1593 (2008), 78-86.

[42] Zhedanov A.S., "Hidden symmetry" of Askey-Wilson polynomials, Theoret. and Math. Phys. 89 (1991), 1146-1157. 